\title{
Die westdeutsche Nebenklagevertretung in den Frankfurter Auschwitz-Prozessen und im Verfahrenskomplex Krumey/Hunsche
}

\begin{abstract}
Der erste Frankfurter Auschwitz-Prozess und seine Wirkungsgeschichte sind im Wesentlichen erforscht. Seine grundlegende Bedeutung für die gesellschaftliche Auseinandersetzung mit der NS-Vergangenheit ist unumstritten. Der Einfluss der Nebenklagevertretung auf Prozessverlauf und-ergebnis ist bislang nicht untersucht. In den Blick genommen werden überdies zwei weitere Verfabren zu nationalsozialistischen Gewalttaten vor dem Frankfurter Landgericht, die bislang im Schatten der Forschung zum ersten AuschwitzProzess standen.
\end{abstract}

Der Umgang mit der NS-Vergangenheit gerät seit Mitte der I $990 e r$ Jahre zunehmend ins Blickfeld historischer Forschung, so auch die justitiellen Bemühungen um Ahndung nationalsozialistischen Unrechts. ${ }^{\mathrm{I}}$ Eine Schlüsselfunktion für die Auseinandersetzung in der bundesrepublikanischen Öffentlichkeit mit der nationalsozialistischen Erblast nimmt hierbei - in außerwissenschaftlicher Wahrnehmung wie in zeitgeschichtlicher Forschung - der erste Frankfurter Auschwitz-Prozess ein: »[...] Wie kaum ein anderes Ereignis der deutschen Nachkriegsgeschichte«, so die ZEIT, habe dieser Prozess »das Selbstverständnis der Bundesrepublik verändert. [...] Nach den Enthüllungen im Frankfurter Prozess « sei »die Identitätssuche der deutschen Demokratie nicht mehr von dem vollständigen Wissen darum zu trennen, dass es Auschwitz gab und was es bedeutete. $\ll^{2}$ Ebenso konstatiert der Historiker Norbert Frei: »Nach dem Prozess, so wird man ohne Übertreibung sagen können, war diesbezüglich nichts mehr so wie vorher.«3

Nicht hinreichend untersucht ist bislang der Einfluss der Verfolgten-Organisationen auf den Komplex der justitiellen Aufarbeitung. Im Folgenden wird ein Gesichtspunkt dieses Komplexes beleuchtet; der Fokus soll auf die westdeutsche Nebenklage, auf die von den Anwälten Henry Ormond und Christian Raabe übernommene Vertretung von Überlebenden und Angehörigen im ersten und

\footnotetext{
I Ein Einstieg: Michael Greve, Der justitielle und rechtspolitische Umgang mit den NS-Gewaltverbrechen in den sechziger Jahren, Frankfurt/Main u.a. 200I, eine Analyse der Urteilspraxis westdeutscher Gerichte: Kerstin Freudiger, Die juristische Aufarbeitung von NS-Verbrechen, Tübingen 2002. Die einzelnen Darstellungen in: Redaktion Kritische Justiz (Hrsg.), Die juristische Aufarbeitung des Unrechts-Staats, Baden-Baden, 1998, ein erster Überblick immer noch bei Viktoria Pollmann, NS-Justiz, Nürnberger Prozesse, NSG-Verfahren. Auswahl-Bibliographie, Frankfurt/Main 2000, s. auch Greve, Neuere Forschungen zu NS-Prozessen. Ein Überblick, KJ I999, S. 472-480.

2 Die Zeit v. I I.I 2.2003, 51/2003.

3 Norbert Frei, Der Frankfurter Auschwitz-Prozess und die deutsche Zeitgeschichtsforschung, in: Fritz Bauer Institut (FBI) (Hrsg.), Auschwitz: Geschichte, Rezeption und Wirkung. Jahrbuch 1996 zur Geschichte und Wirkung des Holocaust, Frankfurt/New York I 996, S. I 23 -I 38, S. I 24.
} 

Hunsche begrenzt bleiben. ${ }^{4}$

Die Nebenklage im ersten Verfahren würdigte Hermann Langbein, Überlebender des Vernichtungslagers und Chronist des Prozesses: »Man kann sich schwer vorstellen, wie der Prozess verlaufen wäre, wenn sich dem Verfahren nicht Nebenkläger angeschlossen hätten. Ein großer Teil der Aufgaben der Anklagebehörde wurde von den Vertretern der Nebenklage übernommen. « $\mathrm{Zu}$ fragen ist hier nach der Bedeutung dieser Nebenklagevertretung für Prozessverlauf und -ergebnis, aber auch nach der gesellschaftlichen und politischen Dimension dieser Opfervertretungen.

\section{Rabmenbedingungen}

Mit der in den frühen I95oer Jahre vorherrschenden Tendenz, die Zeit vor I945 - nicht unbedingt ausschließlich aus apologetischen Gründen - als abgeschlossene Epoche zu betrachten und einen Schlußstrich zu ziehen, ${ }^{6}$ korrespondierten, wie Norbert Frei herausarbeitete, in ihrer Wirkung über die soer Jahre hinausreichende, auch politische Bemühungen um Beendigung, zum Teil um Revision alliierter Entnazifizierungsmaßnahmen. Amnestie- und re-integrations-politische Initiativen »zugunsten eines Millionenheeres ehemaliger Parteigenossen« wurden jedoch von politischer und justitieller »Grenzziehung gegenüber den ideologischen Restgruppen des Nationalsozialismus «lankiert: eine Politik zwischen den Eckpfeilern Amnestie und Integration bei gleichzeitiger »normative[r] Abgrenzung vom Nationalsozialismus «.7 So profitierten von den beiden Straffreiheitsgesetzen von 1949 und $1954^{8}$ und deren teilweise extensiver Auslegung nicht nur Minderbelastete, auch das mit parteiübergreifendem Konsens am

4 Henry Ormond (27.05.190I-08.05.1973), mit 27 Jahren Staatsanwalt, mit 30 Amtsgerichtsrat. Da aus jüdischer Familie stammend, wurde er am 31.5.1933 in den Ruhestand versetzt. Nach KZ-Internierung in Dachau gelang ihm 1939 die Flucht aus Deutschland. Aus seinem englischen Exil kehrte er im Februar I945 als Mitglied einer Sondereinheit nach Deutschland zurück. Ormond war zunächst an Aufbau und Gründung von Tageszeitungen und Zeitschriften - so u.a. des SPIEGEL - beteiligt; zu den biographischen Eckdaten s. Dolf Weber, Henry Ormond - ein juristisches Gewissen Deutschlands, in: Klaus Reichert/Manfred Schiedermair/Albrecht Stockburger/Dolf Weber (Hrsg.), Recht, Geist und Kunst. Liber amicorum für Rüdiger Vollhard, Baden-Baden I996, S.208-224.

5 Hermann Langbein, Der Auschwitz-Prozess. Eine Dokumentation. 2 Bde., Wien I965 Bd. i S.46. (Neuausg. Frankfurt/M. I995). Ebenso Hans Laternser, nicht unumstrittener Verteidiger im Verfahren: »Die zwei Frankfurter Nebenklagevertreter [haben] der Staatsanwaltschaft einen großen Teil der ihr zufallenden Aufgabe abgenommen. "Hans Laternser, Die andere Seite im Auschwitz-Prozess 1963/65, Stuttgart I966, S. 63. Hierzu Christian Dirks, Selekteure als Lebensretter. Die Verteidigungsstrategie des Rechtsanwalts Dr. Hans Laternser, in: FBI (Hrsg.), »Gerichtstag halten über uns selbst...« Geschichte und Wirkung des ersten Frankfurter Auschwitz-Prozesses, Frankfurt/M. u.a. 200I, S. I63-I 92.

6 Exemplarisch Norbert Frei, Amnestiepolitik in den Bonner Anfangsjahren. Die westdeutschen und die NS-Vergangenheit, KJ 1996, S. 484-494, S. 487

7 Für diesen Komplex hat Frei den Begriff »Vergangenheitspolitik «orgeschlagen: Norbert Frei, Vergangenheitspolitik. Die Anfänge der Bundesrepublik und die NS-Vergangenheit, München 1996. Die Zitate S. I 3 f., S. 397; zu den amnestiepol. Weichenstellungen auch Joachim Perels, Amnestien für NS-Täter in der Bundesrepublik, in: Redaktion KJ (Hrsg.), Die juristische Aufarbeitung des Unrechts-Staats, BadenBaden I998, S. 677-687 (zuerst KJ 1995, S. 382-389).

8 BGBl. I949 I, S. 37 u. I954 I, S. 203. Das 2. Gesetz amnestierte in den \$\$ 6 und 9 unter bestimmten Voraussetzungen auch Tötungshandlungen, die in der »Annahme einer Amts-, Dienst-, oder Rechtspflicht « begangen wurden. Zit. n. Perels, Amnestien (Fn. 7), S. 680. Dieser sieht, ebd. S.68 I, hier eine »Tendenz zur Quasi-Amnestie ganzer Gruppen von NS-Verbrechern.« S. a. Frei, Vergangenheitspolitik (Fn. 7), S. 29 ff., S. 100 ff.; Falko Kruse, NS-Prozesse und Restauration, KJ 1978, S. 109-I 34. 
Funktionsträger des NS-Staates « regelte, führte zu einer weitreichenden Wiedereinsetzung entnazifizierter Beamter und damit gerade in den Bereichen der Exekutive und der Justiz zu personeller Kontinuität in nicht geringem Umfang. In diesem Kontext ist ein deutlicher Rückgang der Ahndungstätigkeit nach einer ersten Verfahrenswelle zu sehen, welche kurz nach dem Zusammenbruch ihren Ausgang genommen hatte: Waren zwischen I945 und I95 I noch I7340 Verfahren wegen NS- und Kriegsverbrechen eingeleitet worden, sank die Zahl der Ermittlungsverfahren im darauffolgenden Siebenjahreszeitraum bis I958 auf 2 I32. Die Zahl der rechtskräftigen Verurteilungen ging in dieser zweiten Phase von 5487 auf $467^{10}$ gegenüber der ersten zurück, während die Zahl der Freisprüche oder Verfahrenseinstellungen um Io Prozentpunkte auf $58 \%$ anstieg. ${ }^{\text {I }}$

Neubelebung, organisatorische Systematisierung und Koordinierung erfuhr die strafrechtliche Aufarbeitung ab i 958 durch Etablierung der Zentralen Stelle der Landesjustizverwaltungen zur Aufklärung von NS-Verbrechen in Ludwigsburg. ${ }^{\mathrm{I} 2}$ Impulsgebend hierfür der sog. »Ulmer Einsatzgruppen-Prozess «, ${ }^{\mathrm{I}} 3 \mathrm{der}-$ charakteristisch für die Ermittlungspraxis dieser Phase - durch ein Zusammentreffen von Zufällen in Gang gekommen war und schlaglichtartig Versäumnisse der bisherigen Strafverfolgung offenlegte. Die Kompetenz der Zentralen Stelle war zunächst begrenzt auf das Sammeln und Auswerten verfügbaren Materials und auf Koordinierung der Verfolgung von NS-Verbrechen, die außerhalb der »Altreichs«- Grenzen und nicht unmittelbar in Zusammenhang mit Kriegshandlungen verübt worden waren. Befugnis zur Anklageerhebung hatte die Zentrale Stelle nicht; sobald die Vorermittlungen das Stadium der Anklagereife erreicht hatten, wurde das gesammelte Material an die zuständige Staatsanwaltschaft abgegeben. In dieser Koordinierungsfunktion und zunehmenden Systematisie-

9 Perels, Amnestien (Fn. 7), S. 68I; BGBl. I 1951, S. 307-320; ausführlich Frei, Vergangenheitspolitik (Fn. 7), S. 69 ff.; vgl. Wolfgang Langhorst, Beamtentum und Artikel I 3 I des Grundgesetzes, Frankfurt/ M. I994. Art. I3 I GG besagt, dass die Rechtsverhältnisse der am 8.5.1945 im öffentlichen Dienst Beschäftigten oder Versorgungsberechtigten per Bundesgesetz zu regeln seien. Zur sozialpolitischen Dimension: Hans Günther Hockerts, Sozialpolitische Entscheidungen im Nachkriegsdeutschland. Alliierte und deutsche Sozialversicherungspolitik 1945-1957, Stuttgart 1980. Größenordnung: Von ca. 53000 Entlassenen kehrten ca.52000 in den öffentl. Dienst zurück; Zahlen: Lutz Niethammer, Zum Verhältnis von Reform und Rekonstruktion in der US-Zone am Beispiel der Neuordnung des öffentlichen Dienstes, in: Dietrich Thränhardt u.a. (Hrsg.), Die Bundesrepublik Deutschland. Entstehung, Entwicklung, Struktur, Königstein 1979, S. 47 ff. Perels, Die Restauration der Rechtslehre nach 1945, in: Redaktion KJ (Hrsg.), juristische Aufarbeitung (Fn. I), S. 237 spricht von "struktureller Hypothek. " (zuerst KJ 1984, S. 359379). I 953 waren auf Ministerialebene im AA 40\%, im BMI 42\% »I3 I er«(BA, B I06/7696 \& 7738 , zit. n. Frei, Vergangenheitspolitik (Fn. I), S. 85 ).

Io Die Zahlen: Statistik des BMJ, zit. n. Adalbert Rückerl, Die Strafverfolgung von NS-Verbrechen, I945I978, Karlsruhe 1979, S. I 25. Das Jahr 1948 weist mit 4650 eingeleiteten Verfahren einen Höchstwert aus, danach sinken die Zahlen auf einen Tiefstwert von I 83 in 1954. Ein nennenswerter Anstieg ab I958 (442); (1959: гог 8, I960: 1078). Der Anteil sog. Endphasendelikte ging nach i95 I v. 5I\% d. Strafverfahren auf $26 \%$ zurück (C. F. Rüter/D.W. de Mildt, Die westdeutschen Strafverfahren wegen Nationalsozialistischer Tötungsverbrechen 1945-I997, Amsterdam 1998, S. XI), Das Nachlassen der Ahndungstätigkeit kann also nicht nur hierdurch bedingt sein. Frei, Vergangenheitspolitik (Fn. 7), S. Ioo, spricht hier von »schwersten Unterlassungsschäden [...].« Amnestie- und integrationspolitische Weichenstellungen wird man mit Frei als eine wesentliche Ursache für diese Stagnation sehen müssen.

I I Rüter/de Mildt, Strafverfahren (Fn. Io), S. XI.

I2 Die Zentrale Stelle wurde am I.I2.1958 gegründet aufgrund einer Verwaltungsvereinbarung vom 06.I I.1958. Willi Dreßen, Die Zentrale Stelle der Landesjustizverwaltungen zur Aufklärung von NSVerbrechen in Ludwigsburg, in: Erinnern oder Verweigern. Das schwierige Thema Nationalsozialismus, München I994, S. 85-93, Rüdiger Fleiter, Die Ludwigsburger Zentrale Stelle und ihr politisches und gesellschaftliches Umfeld, in: GWU 53, 2002, S. 32-50.

I 3 Prozess am Schwurgericht Ulm vom 28.4--29.8.1958 geg. Io Angehörige der Gestapo und des SD Tilsit. Hendrik v. Dam/Ralph Giordano (Hrsg.), KZ-Verbrechen vor deutschen Gerichten, Bd. II. Einsatzkommando Tilsit. Der Prozess zu Ulm, Frankfurt/Main, 1966. 
rung der Auswertungs- und Ermittlungstätigkeit liegt die Schlüsselfunktion dieser Institution für die Ahndungstätigkeit der Folgejahre.

Eine gegen Ende der soer Jahre beginnende gesellschaftliche Klimaveränderung, eine durch den Eichmann-Prozess in Israel und dessen Öffentlichkeitswirksamkeit $^{\mathrm{I} 4}$ geförderte Sensibilisierung weiter Kreise in Deutschland, nicht zuletzt die systematische Ermittlungsarbeit der Zentralen Stelle flankieren nun eine mit den I 960er Jahren einsetzende neue Verfahrenswelle ${ }^{\text {Is }}$ mit einer - entsprechend den bis dahin größten Ahndungsdefiziten - Verlagerung der Strafverfolgungsschwerpunkte auf außerhalb der Grenzen der damaligen Bundesrepublik begangene NS-Verbrechen und damit auch auf den Vernichtungslagerkomplex. ${ }^{16}$

Auszumachen ist hierbei die Tendenz, NS-Täter als Gehilfen zu qualifizieren. Unter Rückgriff vor allem auf ein BGH-Urteil ${ }^{17}$ rückt im Rahmen dieser zweiten Prozesswelle die subjektive Teilnahmelehre, die innere Tatbeteiligung des Angeklagten als Entscheidungskriterium zur Abgrenzung von Täterschaft und Beihilfe ins Zentrum der Rechtsprechung unter teils extensiver Auslegung zugunsten der Angeklagten. ${ }^{\text {I }}$

Das Spannungsfeld zwischen einem sich langsam auflösenden, aber immer noch weitreichenden Konsens um Ruhe hinsichtlich des Vergangenen auf der einen und der wachsenden Bereitschaft zur Auseinandersetzung mit der NS-Zeit auf der anderen Seite führte zu Widerständen und Verzögerungen. Strukturelle Gegebenheiten und personelle Kontinuität in Teilen der Justiz- und Exekutivapparate bildeten den Gegenpart zu der systematischen Ermittlungstätigkeit der zentralen Stelle und dem herausragendem Engagement von Einzelpersonen. Die Etablierung von Nebenklagevertretungen in den zu untersuchenden Verfahren kann denn auch nur im Kontext dieser Spannungsfelder betrachtet werden.

\section{Nebenklage im ersten Frankfurter Auschwitz-Prozess}

Der erste Frankfurter Auschwitz-Prozess begann am 20. Dezember 1963 gegen zunächst 22, schließlich 20 Angeklagte. Insgesamt wurden 357 Zeugen gehört,

I4 So auch Norbert Frei, Der Frankfurter Auschwitz Prozess (Fn. 3), S.I 26, dass dieser »nicht eigentlich erst den Wendepunkt [markiert], sondern im Grunde schon die erste Frucht einer Veränderung des vergangenheitspolitischen Klimas « sei.

Is Bereits i959 stieg die Zahl der eingel. Verfahren von 442 (I958) auf Ior 8, im Jahre i960 auf I078, nach zwischenzeitl. Rückgang ein Höchststand I965 ( I 240). Die Zahlen: Rückerl, Strafverfolgung (Fn. I0), S. I 25 .

I6 Zu nennen sind hier die Prozesse z. Treblinka (1964/65), Belzec (1965), Sobibor (1965/66) Verfahrensbeschreibungen bei Rüter/de Mildt, Strafverfahren (Fn. Io), S. XI.

I7 Mit dem sog. »Staschynskij-Urteil« v. I 962 setzte der BGH Maßstäbe für die Rechtsprechung. Staschynskij, KGB-Agent, hatte auf KGB-Befehl zwei Exilpolitiker erschossen; der BGH qualifizierte ihn als Gehilfen, er habe »diese Taten nicht als eigene gewollt, (BGHSt. Bd. I8, S. 87); der BGH schränkte ein, dass derjenige, der »[...] fremde verbrecherische Ziele zur Grundlage eigener Überzeugung und eigenen Handelns macht [...] sich nicht darauf berufen könne, nur Tatgehilfe [...] zu sein. [...]. Er ist regelmäßig Täter.«(ebd.).

I 8 Hierzu Greve, Umgang (Fn. I), v. a. S. I4s ff., Freudiger, Aufarbeitung (Fn. I), passim; dies., Die blokkierte Aufarbeitung von NS-Verbrechen in der Bundesrepublik, in: Joachim Perels u.a. (Hrsg.), NS-Täter in der deutschen Gesellschaft, Hannover 2002, S. I I9-I36, S. i 25 : bei Massenvernichtungsverbrechen in Lagern stehen einer Verurteilung wg. Mordes vier wg. Beihilfe gegenüber, bei Schreibtischverbrechen zeigt sich ein Verhältnis von I:s, bei Stefan Wittke, Teilexkulpation von NS-Verbrechen?, in: Red. KJ (Hrsg.), Die juristische Aufarbeitung (Fn. I), S. 547 ff., für die 6 großen Prozesse (Auschwitz, Chelmno, Bezlzec, Treblinka, Sobibor, Majdanek ) der 6oer- und 70er Jahre - ein Verhältnis von I:3 (S.575); S. a. Perels, Amnestien (Fn. 7), S. 68 5 f. Weiterf. Literatur i. d. Arbeiten in: Red. KJ (Hrsg.) Der Unrechtsstaat, Baden-Baden I983; dies. (Hrsg.), Die juristische Aufarbeitung (Fn. I); z. zeitgenössischen Kritik an d. Tendenz Barbara Just-Dahlmann/Helmut Just, Die Gehilfen. NS-Verbrechen und die Justiz nach I945, Frankfurt/M 1988, Reinhard Henkys (Hrsg.), Die nationalsozialistischen Gewaltverbrechen, Stuttgart 1964 . 

tung für das Zustandekommen des Verfahrens und für die Form, in der es realisiert wurde, war die Initiative des hessischen Generalstaatsanwalts Fritz Bauer, der nach zögerlichen Ermittlungen der Stuttgarter Staatsanwaltschaft das Verfahren an sich zog und eine Übertragung der Zuständigkeit an das Frankfurter Landgericht für alle noch nicht verjährten, in Auschwitz begangenen Straftaten erwirkte. ${ }^{20}$ Bauers Engagement zielte aber auch auf die Grundkonzeption des kommenden Prozesses, indem er auf ein Großverfahren hinwirkte, das den gesamten Komplex der in Auschwitz begangenen Verbrechen aufrollen sollte. ${ }^{21}$ Die Weichen waren im Besonderen durch sein Engagement auf ein in seiner Wirkung über die justitielle Sühneabsicht hinausreichendes Verfahren gestellt.

Mit dem Dritten Strafrechtsänderungsgesetz vom 4.8.1953 gab der Gesetzgeber im Sinne einer Stärkung der Opferrechte auch Hinterbliebenen die Möglichkeit, sich einer öffentlichen Klage anzuschließen. ${ }^{22}$ Vor dem Hintergrund eingangs angesprochener Rahmenbedingungen muss (auch) die Institution der Nebenklage in Verfahren über nationalsozialistische Gewaltverbrechen, in einem wie dem Auschwitz-Prozess konzipierten allemal, zwangsläufig in eine gesellschaftliche, politische, zeitgeschichtliche Dimension ausgreifen. Eine solche Ausrichtung der Nebenklage deutet sich bereits dadurch an, dass in ihrem Zuge keinerlei Entschädigungsansprüche geltend gemacht wurden. ${ }^{23}$

Der Ursprung des Vorhabens, Nebenkläger an einem bevorstehenden Verfahren zu beteiligen, lässt sich nicht klar rekonstruieren; wesentliche Impulse indes, eine solche Korrektivinstanz zu etablieren, gingen von Langbein aus. Spätestens im Januar I960 hatte der damalige Generalsekretär des Internationalen Auschwitz Komitees Kontakt mit Ormond aufgenommen, der sich auch bereit erklärte, in Frage kommende Nebenkläger ohne Honorarforderung zu vertreten. ${ }^{24}$ Richtungsweisend für die künftige Entwicklung der I950 von Ormond in Frankfurt am Main gegründeten Anwaltspraxis und seine weitere Tätigkeit war die noch im selben Jahr erfolgte Übernahme der Klage Norbert Wollheims gegen die

I9 Das Urteil 4 Ks 2/63: C.F. Rüter u.a. (Hrsg.), Justiz und NS-Verbrechen. Sammlung deutscher Strafurteile wegen nationalsozialistischer Tötungsverbrechen, I945-1966, Bd. 22, Amsterdam I98 I. Jetzt auch Friedrich-Martin Balzer/Werner Renz, (Hrsgg.), Das Urteil im Frankfurter Auschwitz-Prozess (19631965), Bonn 2004; Dokumentation: Langbein, Auschwitz-Prozess (Fn. 5); Bernd Naumann, Auschwitz. Bericht über die Strafsache gegen Mulka und andere vor dem Schwurgericht Frankfurt, Frankfurt a.M, Bonn, 1965 (Neuausg: Berlin 2004); Akten: 4 Ks 2/63: HHStA Abt. 46I Nr. 37.638 Bde. I-I29. S. die Beiträge in FBI (Hrsg.), Gerichtstag (Fn. 5); dass. (Hrsg.), Im Labyrinth der Schuld. Täter - Opfer Ankläger, Frankfurt/M. u.a., 2003

20 BGH v. I7.4.1959 (2 Ars 60/59), Akten ${ }_{4} \mathrm{Ks} 2 / 63$, Bl. I ff.; Vgl. Irmtrud Wojak, »Die Mauer des Schweigens durchbrochen. "Der erste Frankfurter Auschwitz-Prozess 1963-1965, in: FBI (Hrsg.), Gerichtstag (Fn. 5), S. $2 \mathrm{I}-42$.

2 I Zu dieser Intention Bauers vgl. ders., Im Namen des Volkes. Die strafrechtliche Bewältigung der Vergangenheit (1965), in: ders, Die Humanität der Rechtsordnung. Ausgewählte Schriften, hgg. von Joachim Perels und Irmtrud Wojak, Frankfurt/Main, 1998, S. 77 ff., sowie Joachim Perels/Irmtrud Wojak, Motive im Denken und Handeln Fritz Bauers, in: ebd., S. 9 ff.

22 Die Befugnisse des Nebenklägers i. d. Paragraphen 395 ff. der StPO, 5. Buch. Er hat die Möglichkeit, Richter, Sachverständige abzulehnen, Frage-, Beweisantrags-, Zeugenbenennungsrecht, das Recht zur Abgabe von Erklärungen, sich zu Schuldfrage und Strafmaß zu äußern, Rechtsmittel einzulegen. Zur strafprozessualen Funktion Claus Roxin, Strafverfahrensrecht, München²5 I998, S. 498 ff.

23 Im Folgenden wird zu untersuchen sein, wie die westdeutsche Nebenklage ihren strafrechtlich gegebenen Spielraum nutzte, um sowohl in den Verfahren selbst, aber auch auf Feldern jenseits der Prozesse Wirkung zu entfalten. Der erste Auschwitz-Prozess wird exemplarisch untersucht, das zweite Verfahren sowie der Verfahrenskomplex Krumey/Hunsche werden zusätzlich für eine Analyse der Rechtsauffassung der Nebenklage herangezogen.

24 »Wir wollen [...] auf dem Weg von Nebenklägern erreichen, dass ein Anwalt sich in das Verfahren einschalten kann. Wir [...] sind [Ormond, C.R.] sehr dankbar dafür, [...] solche Nebenkläger ohne jede Honorarforderung zu vertreten.«, Schr. v. I8.0ı.1960 an URO, in: ÖStA Wien, Nachl. Langbein, E/ı 797:8 5. 
I.G.-Farbenindustrie AG i.L. ${ }^{25}$ Dieser Prozess ist Auftakt einer die Kanzleitätigkeit während der I960er Jahre bestimmenden juristischen Auseinandersetzung mit der nationalsozialistischen Vergangenheit und damit auch Ausgangsbasis der Nebenklagevertretung in den Auschwitz-Prozessen sowie den Krumey/Hunsche-Verfahren.

\section{Im Vorfeld des Prozesses}

Die Voraussetzungen für eine Zulassung zur Nebenklage waren u.a. aufgrund der zeitlichen Distanz zum Geschehen oft nicht leicht zu erfüllen: Zu dokumentieren war das Verwandtschaftsverhältnis zu den in Auschwitz Umgekommenen; überdies war glaubhaft zu machen, dass der Tod der Angehörigen in Zusammenhang mit den den Angeklagten zur Last gelegten Taten stand. Für eine zeitliche Zuordnung genügte hier schließlich der Nachweis des Todeszeitpunkts oder des Deportationsdatums. ${ }^{26}$ Wesentliche Koordinierungsstelle bei der Suche nach geeigneten Nebenklägern war zunächst der World Jewish Congress (WJC). Das Prozedere sah vor, dass der WJC auf seine globale Organisationsstruktur zurückgreifend geeignete Personen ausfindig machen und diese dazu bewegen sollte, Ormonds Kanzlei Prozessvollmacht auszustellen. ${ }^{27} \mathrm{Her}-$ angezogen wurden in der Folge zudem u.a. die United Restitution Organisation (URO), die Conference on Jewish Material Claims against Germany (CJMC), das Nederlands Auschwitz Comité. Nicht zuletzt wegen o.g. Zulassungskriterien, ${ }^{28}$ auch aufgrund einer Reserviertheit vieler potentieller Nebenkläger, sich mit Erlebtem, Tätern, auch deutschen Institutionen zu konfrontieren, ${ }^{29}$ zog sich die Suche nach geeigneten Nebenklägern entsprechend lange hin, so dass bei Eröffnung der gerichtlichen Voruntersuchung am 9.8.196 I ${ }^{30}$ lediglich drei Prozessvollmachten vorlagen. Da die Möglichkeit, Akteneinsicht zu nehmen, Protokolle und Beschlüsse $\mathrm{zu}$ erhalten und sich $\mathrm{zu}$ diesen $\mathrm{zu}$ positionieren, die Zulassung zur Nebenklage voraussetzte, beantragte Ormond diese am 2 I.9.1961. ${ }^{\text {I }}$ Die Zulassung zunächst einer Mandantin und somit die formale Etablierung der Nebenklage erfolgte nun am 23.I0.1961.32 Die Suche nach weiteren Nebenklägern beschleunigte sich: 1962 wurden neun, I963 sechs weitere

25 Hierzu Wolfgang Benz, In Sachen Wollheim gegen I.G. Farben. Von der Feststellungsklage zum Vergleich. Der Frankfurter Lehrprozess, Dachauer Hefte, H. 2, I986, S. 142-174. Wollheim, ehemaliger Zwangsarbeiter der I.G., wollte in einem Musterprozess die Verpflichtung des Konzerns auf Schadensersatz klären lassen. Dieser »test case« endete 1956 in einem vielbeachteten Vergleich zwischen der I.G.Farben i.A. und der CJMC. S. auch Weber, Ormond (Fn. 4), S. 2 I 4 f.

26 Kriterien: Ormond an WJC v. I 5.2.1962, in: FBI, Nachl. Ormond, NL oI-2.

27 Exemplar.: N. Robinson v. WJC an Ormond v. 24.01.1962, in NL oI-2, Allg. Korr.: »Ich wende mich an [...] ehem. Auschwitz Insassen, welche durch uns Fragebogen fuer die Frankfurter Staatsanwaltschaft ausgefuellt haben. Ich hoffe, dass wenigstens einige davon die Vollmacht ausfertigen werden."

28 Exemplarisch: »Da es sehr große Schwierigkeiten macht, Nebenkläger ausfindig zu machen, die gegen einzelne Angeschuldigte zur Nebenklage berechtigt sind, [...]«, Ormond an NAC v. I.2.I962, in NL oI2, Allg. Korr.

29 Die vertrauensbildende Funktion der Organisationen ist zentral: Exemplarisch: WJC an Ormond v. 24.0I.1962: »Es ist leider so, dass [...] es bis heute unmöglich war, durch unsere Verbindungen mehrere Nebenkläger zu finden. Die Menschen sind mißtrauisch, obwohl ich die Verantwortung übernommen hatte, dass ihnen dabei keine Kosten entstehen werden.«, ebd.; möglicher Mandant an Ormond: "[...] teile ich Ihnen höfl. mit, dass ich die Nebenklage [...] nur dann einreichen werde, wenn ich vom Weltkongress direkt aufgefordert werde."

30 Beschluss des LG Frankfurt vom 9.8.196r, Exemplar in Fritz Bauer Institut/Staatliches Museum Auschwitz-Birkenau, Der Auschwitz-Prozess. Tonbandmitschnitte, Protokolle, Dokumente, DVD-ROM, Berlin 2004, S. I.645 f.

3 I Der Antrag in NL OI-2, Allg. Korr.

32 Beschluss v. 23.10.196I, $4 \mathrm{Ks} 2 / 63$, NL OI-2 ebd; 
Sommers 1963 zugelassen. 33 Ormond vertrat bei Prozessbeginn I 5 , schließlich I 4 Nebenkläger aus I 3 Nationen, 34 wobei diese Nebenkläger dem Prozess stellvertretend für die Gesamtheit der aus diesen Ländern stammenden AuschwitzOpfer beigetreten waren. ${ }^{35}$ Sieben dieser Nebenkläger sollten überdies als Zeugen im Prozess gehört werden. Bis auf einen waren sie - I I rassisch, drei politisch Verfolgte - selbst Überlebende des Lagers; 59 Angehörige hatten alle Nebenkläger zusammen in Auschwitz verloren.

Die entscheidenden Impulse zur Realisierung der Nebenklage gingen nun weniger von den Organisationen denn von Ormond selbst aus. Diese beschränkten sich - auch dies oftmals nur auf sein Insistieren - im Wesentlichen auf das erwähnte Prozedere bei der Suche nach geeigneten Nebenklägern sowie auf ihre allerdings nicht zu unterschätzende Bedeutung als Vertrauen stiftende Instanzen angesichts mannigfacher Reserviertheiten möglicher Mandanten. ${ }^{36}$ Hinsichtlich der Implementierung einer Nebenklage-Korrektivinstanz in das kommende Großverfahren treten die Opferorganisationen nicht als entscheidende Antriebskräfte hervor.

Weitaus größere Bedeutung entwickelten sie indes im Rahmen der gerichtlichen Voruntersuchung. Maßgebliche Impulse gingen hier abermals von Langbein aus; so machte er eine nicht geringe Zahl der Zeugen der Anklageseite ausfindig, gab den ermittelnden Behörden zentrale Hinweise und stellte angesichts zunehmender Ost-West-Polarisierung und nicht vorhandener zwischenstaatlicher Beziehungen gleichsam eine außerstaatliche, informelle Plattform für die Kommunikation der deutschen Justizbehörden mit Institutionen und Einzelpersonen (vor allem) in Polen zur Verfügung. ${ }^{37}$

Der WJC leistete - später beginnend als das IAK - vergleichbare Unterstützungsarbeit im Vorfeld des Prozesses, stand allerdings einer Zusammenarbeit mit dem Komitee aufgrund dessen kommunistischen Hintergrundes ${ }^{3}$ äußerst reserviert gegenüber. Man befürchtete, dass die öffentliche Akzeptanz des Verfahrens durch die Unterstützung einer dem Kommunismus nahestehenden Or-

33 Die Beschlüsse v. I 8.7. und 22.7.1963 in Akten 4 Ks 2/63, Bll. I6I68-161 80.

34 Vier der - von Ormond ausgewählten - Nebenkläger wurden auf Vermittlung Langbeins namhaft gemacht, drei über den WJC, einer über besagte belgische Anwaltskanzlei, ein weiterer vom leitenden Untersuchungsrichter, einer über einen bereits zugelassenen Nebenklägers, die Leiterin der Niederländischen Auschwitz Comitees schloss sich der Nebenklage selbst an; ein weiteres Mandat aufgrund Ormonds Privatkontakte, das der restlichen drei ist nicht zu rekonstruieren; vermutl. auch diese über Ormonds Kanzleikontakte. Die Nebenklage richtete sich geg. I7 Angeklagte. Keine Nebenklage war erhoben geg. Bednarek, Schlage und Schoberth. Die Zuordnung der Nebenkläger zu den Angeklagten in $\mathrm{NL} \circ \mathrm{I}-3$.

$35 \mathrm{Zu}$ dieser Konzeption Henry Ormond, Plädoyer im Auschwitz-Prozess (24.05.1965), in: Das Beste aus gestern und heute, Sonderreihe 7 (1965) 63 S., S.2; die Urheberschaft dieser Konzeption ist nicht zu erschließen.

36 Exemplarisch: Ormond an besagte belg. Kanzlei, in NL oI-2: »Nachdem trotz wiederholter Bitten, die ich $[\ldots]$ an [den WJC] gerichtet habe, mir weitere Nebenkläger nicht benannt worden sind, [...] «. Ormond an CJMC v. 9.I.62, ebd.: »Leider ist die Aktion, mir geeignete Nebenkläger [...] zu benennen, derart ins Stocken gekommen, dass mir nichts anderes übrig blieb, als mich selbst nach [...] Persönlichkeiten umzusehen. Das ist [...] nicht der richtige Weg.«

37 Exemplarisch die von Langbein initiierten Kontakte zu Jan Sehn, Beauftragter des polnischen Justizministers: OStA b. Landgericht v. 9.2.1960 an Langbein: »Anliegend darf ich Ihnen ein an Prof. Dr. Jan Sehn gerichtetes Schreiben mit der Bitte um Weitergabe [...] übersenden. " Akten 4 Ks 2/63 Bd. 25, B1. 42 I 5 . In diesem Schreiben an Sehn bat die StA um Kooperation (ebd., Bl. 4216); exemplarisch hinsichtl. israelischer Zeugen: »Können Sie an Frau [...] herantreten, damit von ihr aus ein [...] Protokoll erstellt wird. Es ist mir zwar möglich, ein offizielles Rechtshilfeersuchen an die israelischen Behörden zu richten. Dieser Weg nimmt aber [...] sehr lange Zeit in Anspruch.«, so der zuständige Untersuchungsrichter Dr. Düx in Schreiben v. 20.I 2.196I an Langbein, Akten 4 Ks 2/63, Bll. I0843 f.

$38 \mathrm{Zu}$ Langbeins Distanzierungsprozess v. der KPÖ Anton Pelinka, Langbein und die KPÖ, in: ders., Erika Weinzierl (Hrsg.), Hermann Langbein - Zum 80. Geburtstag, Wien I993, S. 36-39. 
ganisation Schaden nehmen könne, fürchtete eine ideologische Instrumentalisierung des Prozessgegenstandes. 39 Dennoch war der Beitrag der Organisationen im Zuge der Voruntersuchung erheblich, wenn auch ihr Verhältnis untereinander von Misstrauen, politischer und ideologischer Unvereinbarkeit geprägt war. $4^{\circ}$

Ein Niederschlag der Nebenklagevertretung im Ergebnis der Voruntersuchung ist kaum auszumachen: Nach der Zusage Ormonds an das IAK zur Übernahme des Mandats Anfang I 960 liefen einige Zeugenaussagen bei der Kanzlei ein, die an die Staatsanwaltschaft weitergeleitet wurden. . $^{\mathrm{I}}$ Solche Belege sind rar. Mancher Anstoß mag überdies auf informellem Weg von der Kanzlei ausgegangen sein - in diesem Kontext bleibt die Nebenklage weitestgehend von lediglich formaler Bedeutung.

Jedoch gingen von Ormond Impulse im Zusammenhang mit der Konzeption des Verfahrens aus: Zur Erleichterung der Abwicklung des Verfahrens plante Landgerichtsdirektor Hofmeyer eine Aufgliederung des Prozesses in nacheinander zu verhandelnde Einzelkomplexe innerhalb des Gesamtverfahrens, so dass nach und nach immer weitere Angeklagte aus dem Prozess ausgeschieden wären. Ein solches Grundkonzept legte Hofmeyer Ormond in einer Aussprache am 7.II.I963 dar, wogegen Ormond Bedenken formaler Natur geltend machte..$^{2}$ Die ablehnende Haltung des Landgerichtsdirektors gegen ein Verfahrenskonzept, das die Verortung der zur Verhandlung stehenden Tatzusammenhänge in der lagerspezifischen, aber auch der nationalsozialistischen Organisationsstruktur, in ihrem historischen Kontext ermöglichen sollte, war grundsätzlicher Natur: »Die größten prozessualen Schwierigkeiten bieten«, so Hofmeyer auf dem Essener Juristentag I966, »[...] die Großverfahren [...]. Derartige Prozesse sollten [...] unter allen Umständen vermieden werden [...].«43 Eine Abkürzung der jeweiligen Verfahrensdauer angesichts der von der Justiz in dieser Frage noch zu bewältigenden Aufgabe stellte er einer Würdigung des Gesamtgeschehens voran. »[...] Wenn damit die Prozesse von all dem entkleidet werden, was zwar zu den zweifellos historisch interessanten Dingen gehört, so müßte es möglich sein, die Schwierigkeiten dieser Prozesse einzuschränken.«44 Obgleich diese Positionierung des Landgerichtsdirektors bereits vor dem Prozess klar hervortritt, kam es zu dieser »Entkleidung « gleichwohl nicht; wie nun auch immer die von Ormond geäußerten Bedenken hin-

39 Exemplarisch N. Robinson v. WJC an OStA v. I3.01.1960 in: Akten 4 Ks 2/63, Bll. I93 f.: »Persönlich bin ich ueber eine zu große Aktivität des Auschwitz Komitees nicht zu begeistert. Man traut in der Bundesrepublik einer kommunistischen (oder kommunistisch getarnten) Organisation nicht viel. Eine staerkere Identifizierung des Komitees mit dem Verfahren koennte daher der Sache schaedlich sein [...].« N. Robinson an Zentrale Stelle, v. 06.06.1960, in HHStA, Abt. 46I, Nr. 37638, 245, Bl. 427: »[...] Das Comite macht eine große Propaganda für sich [...] ueber den Rahmen seiner Kompetenz (Auschwitz) [sic]. Es waere daher fuer uns sehr wichtig festzustellen, wie gross der Prozentsatz der Wahrheit in deren Behauptungen ist.«

40 Hier nur ein kursorischer Blick auf zwei, in diesem Zusammenhang wesentliche internationale Organisationen. Eine Untersuchung der Bedeutung nicht nur der genannten Organisationen für die Ahndungstätigkeit der deutschen Justiz bleibt Desiderat.

4I So Langbein an die United Restitution Organisation (URO) vom I8.or.1960: »Wir sind ihm sehr dankbar dafür, dass er sich bereit erklärt hat, solche Nebenkläger ohne Honorarforderung zu vertreten. Ich glaube, dass wir uns kaum einen besseren Anwalt hätten wünschen können. « Nachl. Langbein, E/I797: 85. Die Hinweise an StA Frankfurt: Akten 4 Ks 2/63, Bl. 5953 v. 10. 06.1960, Bl. 6279 v. 3.8.1960, Bl. 6084 v. I I.07. 1960; Bd. $36, \mathrm{Bl} 6084$.

42 Gesprächsnotiz Ormonds vom 7.I I.1963 in NL oI-2, Allg. Korr.

43 Hans Hofmeyer, Prozessrechtliche Probleme und praktische Schwierigkeiten bei der Durchführung der Prozesse, in: Verhandlungen des 46. Deutschen Juristentages, Essen I966, Bd. II, Teil C, München u.a. I967, S. $\mathrm{C}_{3} 8-\mathrm{C}_{44}, \mathrm{C}_{39}$.

$44 \mathrm{Zu}$ dieser Prioritätensetzung s. ebd., S. $\mathrm{C}_{4}$ o sowie $\mathrm{C}_{42}$. 
sichtlich der Entscheidungsfindung zu Gunsten des schließlich realisierten

Großverfahrens zu gewichten sind, eine gewisse Impulswirkung wird man so kurz vor Hauptverhandlungsbeginn durchaus veranschlagen können. Die entscheidenden Weichenstellungen dürften aber von der Staatsanwaltschaft vorgenommen worden sein, entsprechend (auch) der konzeptionellen Vorstellung Fritz Bauers.45

\section{Die Zengen der Nebenklage}

Ormond sah nun seine Aufgabe als Vertreter der Nebenklage ${ }^{6} 6$ darin, zum einen in gleicher Front mit der den staatlichen Strafanspruch verfolgenden Staatsanwaltschaft für seine Mandanten »persönliche Genugtuung durch die Bestrafung des schuldigen Täters «47 zu erlangen, aber auch »die Stimme der Opfer zu Gehör $\mathrm{zu}$ bringen ${ }^{48}$ und formulierte damit eine über die juristische Dimension hinausreichende Intention. Eine besondere Notwendigkeit für diese Nebenklage sah er überdies angesichts des "zahlenmäßigen und rhetorischen Übergewicht [s] der Verteidigung.«49

Am 3.8.1964 beantragte Ormond die Ladung von 22 Zeugen, $5^{\circ}$ von denen $20 \mathrm{im}$ Verlauf des Prozesses gehört wurden. Von vermutlich größerem Gewicht als der Niederschlag ihrer Aussagen im Urteils ist ein prozessatmosphärisches, auch dokumentarisches Moment, das mit der Vernehmung dieser Zeugen in den Prozess getragen wurde. ${ }^{2}$ In diesem Kontext sind die Aussagen der beiden letzten auffindbaren und von der Nebenklage benannten Überlebenden des Häftlingssonderkommandos bei den Krematorien und Vergasungsanlagen von herausragender Bedeutung. Die Schilderungen ihrer Tätigkeit blieben nicht ohne - von Ormond intendierter - Wirkung auf die Prozessbeteiligten.53 Welche Bedeutung Ormond dieser prozessatmosphärischen und über den Rahmen des Prozesses hinausgehenden dokumentarischen Komponente für das Verfahren - und damit für die Konzeption der Frankfurter Nebenklage - beimaß, wird in seiner Rückschau auf das Verfahren deutlich: Der Prozess habe der »Mitwelt die Möglichkeit gegeben, die Wahrheit über Auschwitz aus dem Munde der letzten Überlebenden [...] zu hören. Nach ihrem Tod hätte niemand mehr Zeugenschaft

45 Eine wohl nicht allzu weit reichende Kooperation zwischen Bauer und Ormond aufgrund gleichgerichteter Interessen v. a. im Kontext einer Aufklärungsintention: exemplarisch Ormond an Sehn v. I 8. I.1963: »Erst vor kurzer Zeit habe ich Gelegenheit gehabt, mit [...] Dr. Bauer zu sprechen.« [...] Er »möchte mit den modernsten Mitteln und allem zur Verfügung stehenden Anschauungsmaterial die Anklage geführt wissen [...] « Nachlass Sehn, in: Instytut Pamieci Narodowej, Warschau, Sign. Ds.5/63, t.I, ki7, Kopie FBI, NL 03-I.

46 Am ersten Verhandlungstag überreichte Ormond Untervollmacht für seinen Mitarbeiter Christian Raabe, der in der Folge die Nebenklagevertretung gemeinsam mit Ormond wahrnehmen sollte. Anl. Io Prot. 4 Ks 2/63 v. 20.12.1963.

47 Henry Ormond, Plädoyer im Krumey/Hunsche-Verfahren, S. I, NL oI-I 3.

48 Ormond, Plädoyer im Auschwitz-Prozess (Fn. 35), S.r.

49 Drei Staatsanwälten und den Vertretern der Nebenklage standen 17 Verteidiger gegenüber. Henry Ormond, Zwischenbilanz im Auschwitz-Prozess, in: Tribüne, Jg. 3 I964, Heft I I, S. I I 83 -I I90, S. I 87.

50 Antrag vom 3.8.1964, Anl. 4 z. Prot. v. 06.08.1964, 4 Ks 2/63, Akten, Bd. Ior.

5I Die Selektionstätigkeit Klehrs wurde u. a. aufgrund der Aussage eines Nebenklage-Zeugen nachgewiesen (Balzer/Renz, Urteil (Fn. I9), S. 406, S. 4 I I f.), zwei weitere konnten die Authentizität von Beweisunterlagen glaubhaft machen, das Gericht konnte diese so seinen Aussagen zu den allgemeinen Verhältnissen im Lager zugrunde legen; ebd. S. 88, S. 230 und S. 233.; von entsch. Bedeutung für die Verurteilung d. Angekl. Höcker und Stark waren die Aussagen zweier weiterer Nebenklage-Zeugen; ebd., S.I35, S. I 87 ff., S. I 92

52 Die Vernehmungen am 5. und 8.10.1964 DVD (Fn. 30), 20.458, APi78.017 ff.

53 Langbein sah in der Vernehmung dieser Zeugen einen Höhepunkt des Prozesses. Langbein, AuschwitzProzess (Fn. 5), S. 4I. 
ablegen können. In einer nicht fernen Zukunft wäre Auschwitz zur Legende geworden. Dass das nun [...] nicht mehr möglich ist, wird man [...] als das große, das bleibende Verdienst des Frankfurter Auschwitz-Prozesses anzusehen haben.« 54

Verurteilt wurden sechs der verbliebenen 20 Angeklagten wegen Mordes zu lebenslangem Zuchthaus, sieben Angeklagte erhielten wegen Beihilfe zum Mord Zuchthausstrafen von fünf und mehr Jahren, bei vier der Angeklagten lag das Strafmaß nahe der gesetzlichen Mindestgrenze von drei Jahren. Drei der Angeklagten wurden freigesprochen. 55 Raabes Plädoyer im ersten Frankfurter Auschwitz Prozess zu is Angeklagten am I6r. Verhandlungstag ${ }^{56}$ gab Erwägungen zur inneren Tatseite breiten Raum. Er plädierte in elf Fällen auf Mord, in dreien auf Beihilfe, in einem auf Freispruch. Ormond beantragte in seinem Plädoyer zu zwei weiteren Angeklagten, ebenso auf Mord zu erkennen. Die Anträge - exemplarisch, die Angeklagten Mulka und Höcker wegen Mordes in 36.500 bzw. 400.000 Fällen zu 36.500 bzw. 400.000 mal lebenslangem Zuchthaus zu verurteilen - erfahren vor dem Hintergrund, dass die Frankfurter Nebenklage Tatmehrheit zugrunde legte, Plausibilität.

\section{Die Nebenklagevertretung Ostberlins}

Als knapp zwei Wochen vor Hauptverhandlungsbeginn bekannt wurde, dass Ostberlin eine eigenständige Nebenklage im Prozess etablieren werde, hielt Ormond in einer Aktennotiz fest: »Sowohl die Staatsanwaltschaft als auch der Vorsitzende wie auch ich persönlich sind über den neuen Bundesgenossen wenig erfreut, da zu befürchten ist, dass der Prozess als Hintergrund für propagandistische Angriffe dient, und dass die Sache und die Wahrheitsfindung darunter leiden. «s7 Die Entscheidung Ost-Berlins, über das Instrument der Nebenklage im Auschwitz-Prozess Präsenz zu zeigen, muss in der Tat als Element einer Propaganda-Offensive gegen Bonn gesehen werden vor dem Hintergrund einer generellen Zuspitzung des Ost-West-Konflikts und einer sozialen und ökonomischen Krise der DDR. ${ }^{8}$ Die Systemauseinandersetzung, im besonderen in

54 Henry Ormond, Rückblick auf den Auschwitz-Prozess, in: Tribüne, 4. Jg. H. I3, I965, S. I723-1728, S. 1728. In diesem Kontext auch Publikationen und Vorträge Ormonds zw. 1965 und 1969. Ormond wandte sich explizit an die jüngere Generation. Charakteristisch die Fokussierung auf den »Geist von Auschwitz«. Der Prozess findet zumeist nur am Rande Erwähnung. Exemplar.: »Der Geist von Auschwitz«, Ansprache zur Eröffnung der Auschwitz-Ausstellung in Hannover am I7.I I.1965, »Gedanken zum Problem der Schreibtischmörder «, in: Tribüne, Jg. 4, H. I 4 (I965), S. I I I I- I I I7, »Ist der Geist von Auschwitz tot? - Die neue nationalistische Welle«, Vortrag vor der Studentenschaft der pädagogischen Hochschule Hannover am 20.12.1965. Eine Liste der von Ormond verfassten Arbeiten in Nachl. Ormond, ohne Sign.

$5 s$ Kaduk, Baretzki, Boger, Klehr, Bednarek, Stark wg. Mordes, Mulka, Höcker, Schlage, Lucas, Frank, Capesius, Scherpe, Hantl, Dylewski, Broad wg. Beihilfe, freigesprochen die Angeklagten Breitwieser, Schatz und Schoberth; Strafzumessungen s. Balzer/Renz, Urteil (Fn. I9). Mit Ausnahme des Falles Dr. Lucas fand das Urteil Bestätigung i. d. Revision (20.2.1969, 2Str280/67). Zur Revision DVD (Fn. 30), Vorbemerkung zur Strafsache gegen Mulka und andere, S. 58 ff. Keine Nebenklage-Revision war erhoben in den Fällen Schlage, Hantl, Bednarek. Vgl. Ormond an Kurt May v. 6.2.I 969, NL o I-I r. Der honorierte Auftrag zur Wahrung der Interessen der Nebenkläger im Rev.-Verfahren in URO an Ormond v. I0.01.1969, ebd.

56 Raabe plädierte am 21.5.1965 zu Lucas, Frank, Schatz, Breitwieser, Stark, Boger, Dylewski, Hofmann, Kaduk, Klehr, Baretzki, Scherpe, Hantl, Mulka und Höcker (Nachlasss Ormond, FAP-I/NK-II), Ormond am 24.5.1965 zu Broad und Capesius. Henry Ormond, Plädoyer (F. 35). Insgesamt zogen die Nebenkläger eine positive Bilanz des Prozesses, welche Langbein mit Blick auf die Strafmaße nicht teilte. Hinweise auf das hieraus resultierende Zerwürfnis zwischen Langbein und der Kanzlei sind, da beide Seiten Zurückhaltung wahrten, rar. (Vgl. NL ○I-2)

57 Aktenvermerk Ormonds vom 9.I2.1963 über Gespräche mit Staatsanwaltschaft und Hofmeyer, in, NL oI-2, Allg. Korr. 
ihrer innerdeutschen Dimension hinsichtlich des jeweiligen Umgangs mit der nationalsozialistischen Vergangenheit, fand somit Eingang in das Prozessgeschehen.59 Klar hervor tritt hierbei die Intention, die Plattform des Prozesses zu nutzen, um ökonomische Interessen als eigentliche Triebfedern hinter den Auschwitzverbrechen darzustellen sowie die gesellschaftlichen und ökonomischen Strukturen der Bundesrepublik in Kontinuität zu diesen zu setzen. ${ }^{60}$ Diese Grundintention scheint gleichsam als Leitlinie auf in der Nebenklagestrategie des Ostberliner Anwalts Friedrich-Karl Kaul, der die Nebenklage für sechs DDR-Bürger gegen die Angeklagten Mulka, Höcker und Klehr übernommen hatte. ${ }^{61}$ Als Eckpfeiler dieser Strategie zeigen sich Kauls Antrag, ein Gutachten des DDR-Wirtschaftshistorikers Jürgen Kuczynski über »Die Verflechtung von sicherheitspolizeilichen und wirtschaftlichen Interessen bei der Einrichtung und im Betrieb des KZ Auschwitz und seiner Nebenlager « ${ }^{62}$ verlesen zu lassen, ein weiterer Antrag auf Ladung u.a. des DDR-Ministers Erich Markowitsch »zum Beweis dafür, dass das Ausmaß [...] der sogenannten Lager-, Block- bzw. Krankenbauselektionen [...] maßgeblich von den Forderungen der Rüstungsbetriebe bestimmt wurde«, sowie ein weiterer Antrag vom 5.2.1965 auf Vernehmung ehemaliger Mitarbeiter der I.G.-Farben. ${ }^{63}$

Das Gutachten Kuczynskis wurde zwar verlesen, als Beweismittel jedoch nicht anerkannt, da dem Autor nachgewiesen wurde, bei der Auswahl des Quellenmaterials selektiv vorgegangen $\mathrm{zu}$ sein. ${ }^{6}$ Vor der Gutachtenerstattung kam es ein weiteres Mal zum Konflikt zwischen Kaul und Laternser, da letzterer Kuczynski als befangen abgelehnt hatte. ${ }^{65}$ Ormond trat für dessen Anhörung ein und positionierte sich als austarierendes Korrektiv in ideologischer Polarisierung: »Im übrigen finde ich, dass ein bundesrepublikanisches Schwurgericht es verkraften kann, hier einen Sachverständigen zu hören, der gegenwärtig einer anderen Weltanschauung angehört, [...] dieses Gericht kann sich erlauben, tolerant zu sein und die Intoleranz den Gegnern aus der Vergangenheit und aus der Gegen-

58 Vgl. Michael Lemke, Kampagnen gegen Bonn. Die Systemkrise der DDR und die West-Propaganda der SED i $960-1963$, VfZ 1993, S. I $53-174$.

$59 \mathrm{Zu}$ Kaul s. Annette Rosskopf, Friedrich Karl Kaul. Anwalt im geteilten Deutschland (1906-I98I), Berlin 2002.

60 Exemplarisch: »Der Auschwitz-Prozess muss der westdeutschen Bevölkerung vermitteln helfen, dass nur mit Überwindung der Allmacht des [...] Monopolkapitals [...] eine echte Garantie dafür geschaffen werden kann, dass derartige Verbrechen nicht noch einmal den deutschen Namen [...] mit Schande bedecken. "Gustav Hirthe, Bemerkungen zum bisherigen Verlauf des Auschwitz-Prozesses, NJ, Jg. I 8 (1964), S. 305-310.

6I Kaul beantragte Zulassung zunächst für drei DDR-Bürger (Antr. v. 2.I.I964. Prot. 4 Ks/2 63, v. 6.I.1964, Beschluss v. selben Tag ebd.). Am 28.0I. übergab Kaul fünf weitere Vollmachten, drei davon wurden am 31.01.1964 zugelassen.

62 Anl. 4 z. Prot. v. 19.03.1964, 4 Ks 2/63, Bd. 97; dieses war explizit als Gegengutachten zu Broszats Arbeit zum Aufbau der nationalsozialistischen Konzentrationslager konzipiert: Kaul bat darum, dass die Verlesung »unmittelbar nach Abschluss der Befragung des Herrn Dr. Broszat [...]« erfolgen könne. Schr. an Hofmeyer v. 24.02.1964, 4 Ks 2/63, Bl. I7836. Vgl. Florian Schmaltz, Das historische Gutachten Jürgen Kuczynskis zur Rolle der I.G. Farben und des KZ Monowitz im ersten Frankfurter Auschwitz-Prozess, in: FBI (Hrsg.), Gerichtstag (Fn. 5), S. i i 7 ff.

63 Antr. v. 30.07.1964, in NL 0I-3; Markowitsch (1913-199I) war von 1942-1944 Zwangsarbeiter in Buna Monowitz, zur Zeit des Prozesses Mitglied des Ministerrats; Rosskopf, Kaul (Fn. 59), S. 258,; Antrag auf Vernehmung der IG-Mitarbeiter in NL oI-3 ebd.

64 »Die Anträge, [...] Kuczynski als Sachverständigen wegen Besorgnis der Befangenheit abzulehnen, werden für begründet erklärt. «Er habe »eine Reihe von Aussagen der Angehörigen der ehemaligen IG-Farben-Industrie zitiert. Wie er [...] einräumen musste, hat er dabei Aussagen dieser Personen, die sie [...] insbesondere vor dem Nürnberger Militärtribunal gemacht haben, nicht mitverwertet « und es »unterlassen, auf die Existenz solcher weiterer Aussagen [...] hinzuweisen.« DVD (Fn. 30), 19.03.1964 S. 6I37, APoI 9.037

65 Ebd., S. 6r i6, APor 9.00 Iff: »Professor Kuczynski ist nicht unabhängig. [...] Seine Stellung ist davon abhängig, dass er sich [...] bei Erstattung von Gutachten innerhalb der Grundsätze der kommunistischen SED, deren Mitglied er ist, hält.« 
wart zu überlassen. Ich bitte, [...] den Sachverständigen zu hören, und wir werden uns dann alle ein Urteil bilden, ob und welche Schlüsse wir aus diesen Ausführungen [...] ziehen können. «66

Die Einvernahme des Ministers Markowitsch erfolgte am 4.2.1965; der Vorsitzende hielt diese im Wesentlichen auf Fragen nach möglicher Weisungsbefugnis des Stammlagers gegenüber dem Nebenlager Buna-Monowitz - und somit auf die Kompetenzen des Adjutanten Mulka - begrenzt, sie endete freilich im Eklat, als Laternser beantragte, den Minister festzunehmen - es bestehe »der dringende Verdacht «, dass er »an dem Erlaß für den Schießbefehl an der Mauer mitgewirkt « habe. ${ }^{67}$ In diesem Zusammenhang positionierte sich die Frankfurter Nebenklage wiederum als ausgleichende Instanz und widersprach dem Antrag Laternsers, da durch ihn »sachfremde politische Momente in diese Verhandlung hineingetragen werden und die von ihm gestellten Fragen in gar keiner Weise dem ernsten Hintergrund dieses Prozesses entsprechen und in keiner Weise der Wahrheitsfindung [...] dienen können. $\ll^{68}$

Wenngleich der Vorsitzende auch die Vernehmung der I.G.-Mitarbeiter ${ }^{69}$ weitestgehend auf die im Eröffnungsbeschluss definierten Tatkomplexe konzentriert halten konnte und sich keine neuen Erkenntnisse von Beweiskraft ergaben, blieben die Wechselwirkungen zwischen Wirtschaftsinteressen und Lagerbetrieb im Verfahren präsent. ${ }^{\circ}$ Die West-Nebenklage hielt sich in diesem Zusammenhang völlig im Hintergrund. Insgesamt tritt die Frankfurter Nebenklagevertretung als Korrektivinstanz hervor zu einer Instrumentalisierung des Verfahrensgegenstandes in ideologischer Auseinandersetzung - ihre Positionierung gegenüber ihrem Ostberliner Pendant zeigt sich charakterisiert durch ideologische Distanz bei Bereitschaft zu sachorientierter Kooperation.

\section{Inaugenscheinnabme. »Die Erreichbarmachung des unerreichbaren Beweis- mittels«}

Ormond stellte am 8.6.1964 Antrag auf »Augenscheinseinnahme « des Tatorts. ${ }^{71}$ Diplomatische Beziehungen oder Rechtshilfeabkommen zwischen der BRD und Polen bestanden nicht, eine Normalisierung des Verhältnisses schien nicht zuletzt auf Grund der ungelösten Grenzfrage nicht in Sicht. $7^{2}$

Knapp zwei Monate vor Prozessbeginn war Ormond bereits mit der Bitte an Jan Sehn herangetreten, » mit den maßgebenden Instanzen [...] zu klären, ob im Falle eines Lokaltermins in Auschwitz die polnische Regierung innerhalb kürzester Frist die erforderlichen Visen erteilen und die notwendigen Vorkehrungen [...] treffen würde, damit der Termin ungehindert dort stattfinden könnte.« Er habe

66 Ebd. S. 6131, APoi 9.028.

67 Ebd. 04.02.1965, S. $28850, \mathrm{AP}_{272.028 .}$

68 Ebd., S. 28848 , AP272.027.

69 Vernommen wurden zwischen dem 19.2. und dem 9.4.1965 u.a. der ehem. Aufsichtsratsvorsitzende Krauch, die Vorstände Schneider und Bütefisch, der ehem. Werksdirektor Dürrfeld, ebd. unter den jew. Verhandlungstagen.

70 Exemplarisch am 19.02.1965 Hofmeyer hinsichtlich der Vernehmung der IG-Mitarbeiter: Das Gericht habe »diese Zeugen, die Sie [Kaul, C.R.] benannt haben, geladen [...]. Aber nur deshalb, weil Sie [...] eine Beziehung hergestellt haben zwischen den Aussagen der Zeugen und dem heutigen Prozess. DVD (Fn. 30), S.294I 3 f., AP278.053.

7I Antrag: 4 Ks 2/63, Bd. 99, Anl. I z. Prot. v. 8.6.1964.

72 Hierzu Dieter Bingen, Die Polenpolitik der Bonner Republik von Adenauer bis Kohl I949-I991, BadenBaden I 998; Peter Bender, Die »Neue Ostpolitik« und ihre Folgen. Vom Mauerbau bis zur Vereinigung, München ${ }^{4}$ I 996 (Erstausgabe: I 986); Hans-Adolf Jacobsen u.a. (Hrsg.), Bonn-Warschau I945-I 99I. Die deutsch-polnischen Beziehungen. Analyse und Dokumentation, Köln I992. 
Antrag zu stellen.«73 Ormond konnte diesem Antrag schließlich eine Bevollmächtigung Jan Sehns durch den polnischen Justizminister mit der Erklärung beifügen, dass »die Regierung der Volksrepublik Polen dem Gedanken der Abhaltung eines derartigen Lokaltermins positiv « gegenüberstehe und einen entsprechenden Antrag des Gerichts wohlwollend prüfen wolle. Zudem wurde die Bereitschaft signalisiert, »die Einzelpunkte [...] so zu erledigen, dass [...] die Durchführung des Lokalaugenscheins ermöglicht « werde. Diese Bevollmächtigung Sehns habe »ihren Grund in der Überzeugung, dass ein Lokaltermin an der Stätte der Geschehnisse [...] zur Erforschung der objektiven Wahrheit beitragen « werde. ${ }^{74}$ Die Einzelpunkte berührten neben der Visa-Frage auch das Problem einer Souveränitätsverletzung durch die Amtshandlung eines deutschen Gerichts auf polnischem Hoheitsgebiet; auch musste die Zusicherung freien und sicheren Geleits für alle Prozessbeteiligten und damit ein Verzicht polnischer Behörden auf etwaige Strafansprüche gewährleistet sein. Diese Fragen hatte Ormond mit Schreiben vom 3.2.1964 präzisiert75 - die Kooperationsbereitschaft Polens hatte detaillierte Bezugspunkte. ${ }^{76}$ Dass die Augenscheinseinnahme auf der Basis einer Übereinkunft zwischen dem Vorsitzenden des Gerichts und dem Justizminister Polens erfolgte und nicht aufgrund einer zwischenstaatlichen Vereinbarung, wirft angesichts der Kooperationsbereitschaft Polens ein erstes Schlaglicht auf die Positionierung der Bundesregierung hierbei. Diese ist charakterisiert durch Bestrebungen, die Frage der Inaugenscheinnahme auf zu klärende "juristische Detailfragen " zu reduzieren und in den Verantwortungsbereich der Justizbehörden zu delegieren.77 Am 20.10.1964 beschloss nun das Gericht, den Ortstermin kommissarisch durch ein richterliches Mitglied des Schwurgerichts durchführen zu lassen. $7^{7}$ Die Schwierigkeit, die in Haft befindlichen Angeklagten ins Ausland verbringen zu müssen und damit Revisionsgründe zu schaffen, wurde somit umgangen, die Beanspruchung polnischer Souveränität gleichzeitig begrenzt. Am 2.I2.I964 gab der polnische Justizminister die »Einwilligung zur Vornahme einer Ortsbesichtigung [...] als einer Prozesshandlung im Rahmen

73 Jan Sehn (1909 -1965) fungierte in den Krakauer Auschwitz-Prozessen I 947/48 als Untersuchungsrichter, leitete ab 1949 das Kriminologische Institut in Warschau, bezügl. des Frankfurter Prozesses war er Bevollmächtigter des polnischen Justizministeriums. Beide Zitate Ormond an Sehn v. 17.10.1963, in: Nachlaß Sehn, Ds.5/63, tı, k.36. Erste Kontakte Ormonds zu Sehn sind für Februar I 962 nachzuweisen. Vermerk Ormonds v. 28.2.1962, NL oI-2, Allg. Korr.

74 Schreiben des poln. Ministers der Justiz, 4 Ks 2/63, Anl. I z. Prot. v. o8.06.1964.

75 Ormond an Sehn, Nachlaß Sehn Ds.5/63, t.I, k.47. Im Zentrum stehen die Frage nach Visa-Erteilung, Exekutivgewalt, Begleitung durch deutsche Polizeibeamte, sicheren Gewahrsams der inhaftierten Angeklagten, deren sicheres Geleit. Antragsbegründung: Er sei zuversichtlich, dass diese »Schwierigkeiten bei gutem Willen auf deutscher und auf polnischer Seite überwunden werden können«. Auschwitz-Prozess, DVD-ROM (Fn. 30), APo6r.t43.

76 Die Souveränitätsfrage stand im Mittelpunkt der Auseinandersetzung im Verfahren: Der Vorsitzende artikulierte hier größte Bedenken (ebd., t44), bei der Verteidigung keine einheitliche Linie. Verteidiger Schallock beantragte, Ormonds Antrag zuzustimmen, Laternser lehnte ihn als unsubstantiiert ab, ebd., APo61.t49; Laternsers Einwand AP076.tIo. Kaul schloß sich Ormonds Antrag am i r.6. an, die Staatsanwaltschaft am 22.06. ${ }_{4}$ Ks 2/63, Bd. 99, Anl. 2 z. Prot. v. I I.06.1964 und Anl. I z. Prot. v. 22.06. Ormond substantiierte seinen Antrag am 30.06. Ebd. Bd. 100, Anl. 2 z. Prot. v. 03.07.

77 Hierzu Sybille Steinbacher, »Protokoll vor der Schwarzen Wand.« Die Ortsbesichtigung des Frankfurter Schwurgerichts in Auschwitz, in: FBI (Hrsg.), Gerichtstag (Fn. 5), S. 6I-96: »Die Bundesregierung konnte sich am Ende allenfalls auf die Fahnen schreiben, die Ortsbesichtigung [...] nicht nachdrücklich behindert zu haben. «, ebd., S. 7 I f. Steinbacher wertet v.a. Unterlagen des Bundeskanzleramtes und BMJ aus, Material des Hessischen Justizministeriums zieht heran Werner Renz, Tatort Auschwitz. Ortstermin im Auschwitz-Prozess, in: Tribüne I 58/200I, S. I32-I44, diese Untersuchung wertet ergänzendes Material aus den Prozessunterlagen sowie den Nachlässen Ormonds und Sehns aus.

78 Vgl. Schreiben Hofmeyers an den polnischen Minister der Justiz vom 27.10.1964, in: 4 Ks 2/63, Bll. I 8 I 5 I ff. 
des Beweisverfahrens.«79 Die Ortsbesichtigung wurde gemäß der deutschen Strafprozessordnung zwischen dem I4. und I6.I2.I964 ${ }^{80}$ durchgeführt; Sehn übertrug dem beauftragten Richter die Sitzungspolizeigewalt - polnische Exekutivorgane standen in der Verfügungsgewalt bundesdeutscher Judikative.

Nach Abschluss der Ortsbesichtigung traf der beauftragte Richter mit dem polnischen Justizminister zusammen, obgleich das Zustandekommen eines solchen Empfangs bis zuletzt unsicher geblieben war angesichts einer Verstimmung der polnischen Seite darüber, dass Sehn selbst alle Verhandlungen mit dem Vorsitzenden des Schwurgerichts zu führen hatte und nie - ein weiteres Schlaglicht auf die Haltung Bonns - auf Ministerialebene empfangen worden war. ${ }^{8 \mathrm{I}}$ Wenngleich die im Vorfeld des Prozesses etablierten Kontakte zwischen Staatsanwaltschaft und polnischen Behörden für Ormonds Initiative zweifellos von Nutzen waren, ist die Realisierung dieses Ortstermins auf sein und Jan Sehns Engagement zurückzuführen. Für den weiteren Prozessverlauf war die Ortsbesichtigung von einiger Bedeutung. Sie gab dem Schwurgericht »eine festere Grundlage für sein Urteil ${ }^{82}$ indem sie zur Klärung räumlicher, akustischer Gegebenheiten und Sichtmöglichkeiten beitrug. Ein Großteil der Einlassungen der Angeklagten sowie der Aussagen von Zeugen der Verteidigung konnten widerlegt werden, die meisten Bekundungen der Anklagezeugen fanden durch den Ortstermin hingegen Bestätigung. ${ }^{8} 3$ Überdies kommt hier eine von Ormond beabsichtigte, »nachhaltige Wirkung ${ }^{8}{ }^{4}$ der Konfrontation mit dem Tatort Auschwitz auf die Prozessbeteiligten zum Tragen, welche zweifellos Kriterium bei der Stellung des Antrags gewesen war; hinsichtlich seiner Erwartungen führte Ormond aus, »dass für das Verständnis der Berufsrichter und der Geschworenen ein solcher Termin [...] sich nur günstig auswirken könnte, da die Prozessbeteiligten sich dem Eindruck von Auschwitz nicht entziehen könnten « 85 - womit wiederum eine auf die Prozessatmosphäre zielende Komponente der Nebenklagevertretung, die Intention, die Realität des Lagers im Prozess wirkmächtig werden zu lassen, klar hervortritt. Der Ortstermin leistete keinen geringen Beitrag zur Wahrheitsfindung. Er trug durch sein weltweites Medienecho - etwa I o० Journalisten aus aller Welt begleiteten die Delegation ${ }^{86}$ - dazu bei, die Wirklichkeit Auschwitz näher an die bundesrepublikanische Gegenwart der i $960 e r$ Jahre heranzuholen.

79 Justizminister Polens an Hofmeyer vom 2.I2.1964 (begl. Übersetzung), 4 Ks 2/63, Bll. I8297. Am I 2.I 2.I 964 reisten auf dieser Basis neben dem beauftragten Richter 23 Personen nach Polen. So u.a. zwei Justizwachtmeister, drei Vertreter der Staatsanwaltschaft, die Nebenklagevertreter, ein Nebenkläger, elf Verteidiger sowie als einziger Angeklagter Dr. Lucas. Vgl. hierzu das Prot. d. beauftr. Richters Hotz a. d. BMJ, 4 Ks 2/63, Bll. I 8294 ff. Die folgenden Details ebd.

80 Vgl. d. Bericht d. Amtsgerichtsrats Hotz über die Inaugenscheinnahme a. d. Bundesjustizminister v. 5.I.1965, Akten 4 Ks 2/63, Bll. I 8294 ff.

8 I Ebd. Bl. I 8298. Ormond und Raabe wurden von Ministerpräsident Cyrankiewicz empfangen - bei diesem, selbst Überlebender des Lagers, liegt wohl auch der Schlüssel für das Verständnis der polnischen Kooperationsbereitschaft. Sehn äußerte vor Abflug der Delegation, diese Ortsbesichtigung sei ein Präzedenzfall gewesen - und somit »in der Lage, Wege zwischen Staaten und Völkern zu ebnen. Zit. n. ebd., S. 7 (Bl. I 8300). Präzedenz jedenfalls für drei weitere Ortstermine in westdeutschen Strafverfahren. Vgl. hierzu Steinbacher, Protokoll (Fn. 77), S. 80.

82 »Aus Auschwitz zurück «, FAZ v. 22.1 2.64, zit. n. Steinbacher, Protokoll (Fn. 77), S. 88,

83 Vgl. die zahlreichen Bezugnahmen auf den Ortstermin im Plädoyer des Staatsanwalts Vogel am 07.05.1965. Auschwitz-Prozess, DVD-ROM (Fn. 30), S. 32918, AP367.060.

84 Renz, Tatort Auschwitz (Fn. 77), S. I40, vgl. hierzu auch Steinbacher (Fn. 77), Protokoll, S. 77.

85 Ormond an Sehn v. I7.10.1963, in: Nachlaß Sehn, Ds.5/63, t.I, k.36.

86 Die Schätzung bei Hotz, Bericht, S. 2; s.a. Steinbacher, Protokoll (Fn. 77), S. 75 f. 
»Im Gegensatz zum ersten Prozess «, so Hannah Arendt, »wurde über den zweiten in der Presse derart wenig berichtet, dass es einiger $>$ Nachforschungen bedurfte, um festzustellen, ob er überhaupt stattgefunden hatte. $\ll^{87}$ Die mangelnde Wahrnehmung des zweiten Frankfurter Auschwitz-Prozesses findet ihre Entsprechung in der Forschung - das knapp neun Monate dauernde Verfahren ist bislang nicht untersucht worden. ${ }^{88}$ Nicht zuletzt die hier verhandelten Tatkomplexe machen jedoch deutlich, dass es sich bei diesem Verfahren um mehr als eine justitielle Nachlese des ersten handelt. Standen im ersten Prozess neben den zwei Adjutanten der Kommandantur im Wesentlichen an letzter Stelle des Vernichtungsprozesses Ausführende vor Gericht, ${ }^{89}$ verschob sich im zweiten der Fokus entsprechend der früheren Funktionen der drei Angeklagten auf die Verwaltungskomponente des Vernichtungslagers sowie auf mit dem Außenlager Buna-Monowitz zusammenhängende Tatkomplexe. Hierdurch waren in weit größerem Maße als im ersten Prozess Berührungspunkte zur I.G.-Farben-Problematik gegeben.

Der Angeklagte Burger war von Juli I 942 bis April I 943 Leiter der Verwaltung des Vernichtungslagers und in dieser Eigenschaft u.a. für die Beschaffung des Zyklon B verantwortlich; Josef Erber hatte von Oktober 1943 bis April 1944 die Position des Leiters der Aufnahmeabteilung des Lagers inne und war angeklagt, in dieser Funktion an Rampenselektionen teilgenommen zu haben. Neubert schließlich war von 1943 bis zur Evakuierung des Lagers am I 8. I. 945 als Sanitätsdienstgrad im Lager Monowitz und hatte sich wegen Selektionen im Häftlingskrankenbau zu verantworten. $9^{\circ}$

Mit den ebenso in Frankfurt verhandelten Verfahren gegen Hermann Krumey und Otto Hunsche, beide Mitarbeiter an maßgeblicher Stelle in Eichmanns Sondereinsatzkommando in Ungarn, gerät eine weitere Facette des Zusammenhangs, der organisatorisch-»logistische « Aspekt der Deportation, ins Blickfeld. Die Tatkomplexe dieser drei Verfahren treten als sich ergänzende Komponenten im Vernichtungszusammenhang Auschwitz hervor; nicht zuletzt deshalb bietet es sich an - auch vor dem Hintergrund der Intention Fritz Bauers, den gesamten Vernichtungskomplex zum Verfahrensgegenstand zu machen -, den zweiten Frankfurter Prozess und das Verfahren Krumey/Hunsche stärker in die Forschung zum großen Auschwitz-Prozess einzubeziehen, die drei Verfahren als Komplementär-Prozesse zu betrachten hinsichtlich der Geschichte der strafrechtlichen Bemühungen um Aufarbeitung des Komplexes Auschwitz durch das hierfür zuständige Frankfurter Landgericht.

Wenngleich die Frankfurter Anklagebehörde seit Mitte der soer Jahre auch für den Ungarnkomplex zuständig war, ${ }^{91}$ scheinen hier weniger »Masterpläne « der Anklagebehörde, sondern die Bedingungen staatsanwaltschaftlicher Ermittlung,

87 Hannah Arendt, Der Auschwitz-Prozess, in: dies., Nach Auschwitz. Essays \& Kommentare I, hrsgg. v. Eike Geisel u. a., Berlin 1989, S. 99-136, S.99.

$88{ }_{4} \mathrm{Ks} 3 / 63$ (I4.I 2.1965 - I6.9. I 966), HHStA, Abt. 46I I 2/2002. Rosskopf, Kaul (Fn. 59), S. 269 ff. erwähnt das Verfahren kurz. Vgl. Christian Dirks, Wie die DDR ihr eigenes Tribunal inszenierte, in: Frankfurter Rundschau, 4.10.200I, S. 9 ff.

89 An Selektionen beteiligte SS-Ärzte, v. a. niedere Dienstgrade aus der pol. Abt., der Schutzhaftlagerführung, des Häftlingskrankenbaus, ein Funktionshäftling. Zur Funktion vgl. die Lebensläufe in Balzer/ Renz, Urteil (Fn. I9), S. 89 ff.

90 Die vorst. Angaben in: Urteil $4 \mathrm{Ks}_{3} / 6_{3}$, in NL o I-I3, S. 5 I ff., und S. $96 \mathrm{ff}$.

9i Vgl. Fritz Bauer, Im Namen des Volkes. Die strafrechtliche Bewältigung der Vergangenheit (1965), in: ders., Humanität, S. 77-90, S.8 I; vgl. auch Mathias Meusch, Von der Diktatur zur Demokratie: Fritz Bauer und die Aufarbeitung der NS-Verbrechen in Hessen (1956-1968), Wiesbaden 200I, vor allem S. I 82. 
die Verfügbarkeit beweisrelevanten Materials, aber auch Zufälle ursächlich zu sein für die Konstellation der Komplementärprozesse. $9^{2}$

\section{Der Verfabrenskomplex Krumey/Hunsche}

Zeitgleich mit der Besetzung Ungarns ab dem I9.3.I944 rückte auch Eichmanns Sondereinsatzkommando (SEK) ein und konstituierte sich am 4. oder 5 . Tag der Besetzung formell. 93 Die einzige Aufgabe des SEK bestand darin, die ungarischen Juden aus dem öffentlichen Leben auszuschalten, zu konzentrieren und schließlich zu deportieren. Seine Weisungen erhielt das Kommando ausschließlich vom RSHA. Binnen weniger Wochen nach dem I 5.5. I 944 wurden zwischen 434000 und 438000 jüdische Menschen abtransportiert, von denen fast alle nach Auschwitz kamen.

Dem Juristen Otto Hunsche oblag der Kontakt zum ungarischen Innenministerium. Nach Ansicht des Schwurgerichts im zweiten Verfahren I 969 verkörperte er dem Ministerium gegenüber, das die Judengesetzgebung selbst vollzog, die Besatzungsmacht, er unterband durch seine Präsenz Verzögerungen von Anfang an. 94

Hermann Krumey, als Obersturmbannführer im gleichen Dienstrang wie Eichmann, fungierte im Rahmen des SEK als dessen Vertreter, stand dem gesamten inneren Betrieb der Dienststelle vor, wirkte hier an entscheidender Stelle mit, die organisatorischen Grundlagen für den reibungslosen Ablauf der Aktion zu legen; überdies war er für alle Verhandlungen mit Vertretern der ungarischen Juden zuständig und somit wesentlich an der Verschleierung des eigentlichen Zwecks der Aktion beteiligt.95

Ein erstes Urteil gegen Hunsche erging am I3. Juli i 962. In diesem Verfahren, dem sich ein Mitglied des Weltverbandes der ungarischen Juden als Nebenkläger angeschlossen hatte, ${ }^{96}$ wurde Hunsche zu 5 Jahren Zuchthaus verurteilt. ${ }^{77}$ Der 2. Strafsenat des Bundesgerichtshofs hob dieses Urteil aufgrund der Revision von Staatsanwaltschaft und Nebenklage am 20. Mai 1963 wieder auf. $9^{8}$ Dieses

92 Exemplarisch die Sanitätsdienstgrad-Tätigkeit des Angeklagten Neubert, welche ursprünglich im ersten Prozess verhandelt werden sollte; das Verfahren wurde wegen Verhandlungsunfähigkeit Neuberts abgetrennt - dieser Tatkomplex wurde somit ledigl. aus prozessrechtlichen Gründen im zweiten Verfahren verhandelt.

93 Das Verfahren hat Freudiger, Aufarbeitung (Fn. I), S.97 ff., in ihre Urteilsanalyse einbezogen, hier ein kurzer Überblick. Die folgenden Angaben zur Funktion des SEK im Revisionsurteil 2 Str 279/66, Exemplar NL oI-19, S. 3 f. Die Akten: 4 Ks I/63: HHStA, Abt. 46I Nr. 33.53 I-33.75 I.

94 Die voranstehenden Angaben in: Urteilsbegr. ${ }_{4} \mathrm{Ksi} / 63$, I969, Exemplar NL oI-19, S. 95 f. Hunsche war ab Januar I 940 Mitarbeiter der Gestapo. Am 29.I I.I94I wurde er ins Dezernat IV B 4 des RSHA versetzt und war dort mit Staatsangehörigkeits- und Vermögensfragen bereits deportierter Juden befasst. Am I4.IO.I947 (Akt.Z. SpLs 90/47) wurde er wegen Gestapozugehörigkeit zu zwei Jahren und drei Monaten Gefängnis unter Anrechnung der Internierungshaft verurteilt. Ab 17.8.1948 auf freiem Fuß, wurde er als Mitläufer eingestuft. I956 erreichte er die Zulassung als Rechtsanwalt. Bis zu seiner Verhaftung I 960 betrieb er eine Kanzlei. Untersuchungshaft v. 23.5.1957 bis I 8.6.1957, vom I 8.1 I.I960 bis 8.2.1963 sowie vom 25.4.1964 bis 3.2.1965.

95 Die vorstehenden Angaben in: Urteilsbegr. ${ }_{4} \mathrm{Ks}$ I/63, I969, S. 96 ff. Ab März I 940 war Krumey Leiter der »Umwandererzentrale Posen« und verantwortlich für die Umsiedelung der »Fremdvölkischen« im Warthegau sowie deren Auslese. Krumey habe hierbei völlig freie Hand gehabt - bis April 1943 wurden über 600000 Personen ausgesiedelt und teilw. zum »Arbeitseinsatz « verbracht. I947 wurde er als Mitläufer eingestuft. Untersuchungshaft vom I.4.1957 bis 18.6.1957, vom 24.5.1960 bis 10.2.1965 und ab 24.I.I969 bis 29.8.1969.

96 Vgl. Rev.-Urteil 2 StR 594/62 v. 20.05.1963 zu 4 Ks I/62, S. 4.

97 Die Akten 4 Ks I/62 im HHStA Wiesbaden, Abt 46I.

98 Mit Rev.-Urteil 2 StR 594/62. Die Revision der Anklage richtete sich gegen die günstige Bewertung der subjektiven Teilnahme des Angeklagten am Vernichtungsgeschehen und damit gegen dessen Verurteilung wegen Beihilfe. 
somit an das Frankfurter Landgericht zurückverwiesene Verfahren wurde nun mit jenem gegen Hermann Krumey verbunden.99 Krumey wurde hier der Beihilfe zum Mord in mindestens 300.000 Fällen für schuldig befunden und zu einer Zuchthausstrafe von s Jahren verurteilt, Hunsche indes freigesprochen. Das Gericht war zwar davon ausgegangen, dass die »Durchführung der Massenvernichtung der ungarischen Juden auf einem vorher gefassten Gesamtplan « beruht habe und daher Handlungseinheit vorliege, den Beginn der einheitlichen Handlung des Vernichtungsvorgangs sah das Gericht jedoch erst in der Verladung der Opfer in die Deportationszüge, während alle Handlungen im Vorfeld dieses Punktes als »Vorbereitungshandlungen « zu werten seien. ${ }^{100}$ Das Gericht klammerte alle organisatorischen Maßnahmen vor der eigentlichen Deportationshandlung aus dem Vernichtungsvorgang aus.

Das Gericht im Revisionsverfahren ${ }^{\mathrm{IOI}}$ hierzu sah es indes als erwiesen an, dass Krumey an der Tötung von mindestens 290000 Menschen aus Rassenhass, damit aus niedrigen Beweggründen, mitgewirkt habe. Die gesamte Vernichtungsaktion der ungarischen Juden wurde hier nun tateinheitlich aufgefasst. Bei dieser einen Tat sei Krumey Mittäter gewesen. Krumey habe um die Tötung der Juden gewusst und diese gewollt. Bei Hunsche hingegen erkannte das Schwurgericht keinen Täterwillen und entschied damit auf Beihilfe. ${ }^{102}$ Über diesen Verfahrenskomplex tritt die beim justitiellen Umgang mit sogenannten »Schreibtischtätern« zentrale Problematik deutlich hervor - mit zunehmender Distanz des Tatbeteiligten zur letztendlichen Tötungshandlung gewinnt die Abgrenzung einer einheitlichen Tat an Bedeutung. Diese Problematik sollte sich als zentral erweisen für Positionsverschiebungen in der Rechtsauffassung der Frankfurter Nebenklagevertretung. ${ }^{103}$

\section{Der zweite Frankfurter Auschwitz-Prozess}

Ursprünglich war nicht daran gedacht, Nebenkläger im zweiten AuschwitzProzess aufzustellen. Vielmehr hatte Ormond mit dem WJC vereinbart, künftige Nebenklagen auf zeitgeschichtlich relevantere Prozesse zu konzentrieren; als solche betrachtete man vorrangig das in Hagen anhängige Verfahren bezüglich des Vernichtungslagers Sobibor, den Prozess zum Warschauer Ghetto in Hamburg sowie Prozesse gegen Beamte des RSHA im Allgemeinen ${ }^{104}$ - womit ein weiteres mal die Bedeutung der Komponente der Öffentlichkeitswirksamkeit, der Bemühungen um gesellschaftliche Aufklärung in der Nebenklagekonzeption deutlich wird.

99 Urteil 4 Ks I/63 v. 3.2.1965, HHStA Wiesbaden, zit. n. NL oI-19.

Io० Beide Zitate ebd. Nebenklage und Staatsanwaltschaft hatten Täterschaft angenommen. Diese Argumentationslinie bereits im ersten Hunsche-Verfahren: Hunsche habe aus »zeitlichen und räumlichen Gründen keinen Einfluss im Sinne der Tatherrschaft auf die Durchführung der geplanten Tötung der in das Konzentrationslager Auschwitz verbrachten jüdischen Personen« gehabt.

Ior Urteil 4 Ks I/63 vom 29.8.1969., in: NL OI-I 9

I02 Die voranstehenden Angaben ebd., S. I4I ff. und S. I44.

I03 Im ersten gegen beide Angeklagte geführten Verfahren hatte die Frankfurter Kanzlei zwei Vertreter des Weltverbandes der ungarischen Juden vertreten (vgl. Plädoyer Ormonds im Krumey/Hunsche-Verfahren, in NL oI-I9, S. I). Die gesamte Bandbreite der Formen justitiellen Umgangs mit »Distanztätern « finden sich in diesem das Landgericht knapp I 3 Jahre beschäftigenden Verfahrenskomplex. Für eine exemplarische Untersuchung böte er sich daher an.

I०4 Diese Vorgehensweise vereinbarten Ormond und Jacob Robinson, Direktor des Institute for Jewish Affairs und Berater der Anklagebehörde im Eichmann-Prozess am I0.09.1965. Aktenvermerk Ormonds NL oI-I3, Allg. Korr. 
Ausschlaggebend für den Entschluss, entgegen dieser Prioritätensetzung nun doch im zweiten Verfahren präsent zu sein, war die Ankündigung einer wiederum von Kaul geführten Nebenklagevertretung Ost-Berlins, ${ }^{105}$ wobei zu erwarten stand, dass die Verbindung zwischen dem I.G.-Farben-Komplex und dem Vernichtungslager über die Tätigkeit Neuberts als Sanitätsdienstgrad in Monowitz eine stärkere Thematisierung erfahren würde. Als entscheidendes Kriterium für die Etablierung einer Frankfurter Nebenklage tritt nun hervor, hinsichtlich dieses Themenkomplexes ein Korrektiv zur Ostberliner Opfervertretung zu schaffen. Tritt die Korrektivfunktion hinsichtlich der ideologischen Instrumentalisierung des Prozessgegenstandes in der Nebenklagekonzeption bereits im ersten Prozess zu Tage, ist dieser Faktor im zweiten konstitutiv für ihre Etablierung. ${ }^{106}$

Verurteilt wurde Burger wegen Beihilfe zum gemeinschaftlichen Mord zu 8 Jahren Zuchthaus, verbüßt durch eine in Polen vollzogene Gefängnisstrafe. ${ }^{107} \mathrm{Als}$ erwiesen sah das Gericht an, dass das »benötigte Giftgas Zyklon B ausschließlich vom Angeklagten oder von den Angehörigen der ihm unmittelbar unterstehenden Verwaltung bestellt und bezahlt wurde.«108 Das Gericht ging hierbei, ebenso wie Staatsanwaltschaft und Nebenklage, von Tateinheit aus, wertete aber nicht die »Endlösung « als einheitliche Tat, sondern betrachtete jede einzelne Vernichtungsaktion als eine Handlung im Rechtssinne, an der »eine Vielzahl von Tätern und Gehilfen « - Burger durch die Gasbestellungen - mitgewirkt hätten. ${ }^{109}$ Die subjektive Teilnahme Burgers war allerdings auch der Punkt, an dem sich die Geister schieden: Die Frankfurter Nebenklage nahm Täterschaft an und beantragte lebenslanges Zuchthaus; Raabe ging davon aus, Burger habe seinen »Tatbeitrag in Auschwitz mit [...] vollem Einverständnis mit dem nationalsozialistischen Vernichtungsprogramm« geleistet - ein Mann mit seinem Werdegang sei »kein widerwillig handelnder Gehilfe" gewesen. ${ }^{110}$

Erber wurde wegen gemeinschaftlichen Mordes in 70 Fällen zu einer lebenslangen Zuchthausstrafe verurteilt - das Gericht war hierbei den Anträgen der Anklagebehörde und der Nebenklage gefolgt. Ihm konnte die Teilnahme an Selektionen an der Rampe sowie im Lager selbst nachgewiesen werden, ebenso betrachtete das Gericht seine Teilnahme an Erschießungen als erwiesen. Er habe dabei aus niedrigen Beweggründen gehandelt, »die Anschauung derer, die das Vernichtungsvorhaben geplant und befohlen hatten«, geteilt. Er habe Befehle ausgeführt, die er innerlich bejaht habe. ${ }^{\text {II }}$

Ios Zur Entscheidungsfindung vgl. Ormond an URO v. 23.09.1965, in: ebd: »Als Professor Robinson hier war, sprachen wir auch über die Frage der Nebenklagevertretung im zweiten Auschwitzprozess. Ich hielt sie nicht für erforderlich. Professor Robinson stimmte dem zu. [...] Nun höre ich aber, dass Professor Kaul, der im Fall Neubert (Buna-Komplex!) als Nebenkläger zugelassen wurde, wieder erscheinen wird. Wir müßten [...] klären, ob bei dieser Sachlage eine Vertretung von unserer Seite doch erfolgen soll.«

I06 Am 25.r I. I965 übertrug die URO der Kanzlei das Mandat. Vgl. Ormond an URO v. 3.I 2.1965, ebd. Ormond hielt sich auf Grund der aus dem ersten Verfahren resultierenden Arbeitsüberlastung - so die offizielle Begründung - im Hintergrund. Vgl. Schreiben Raabes an Mariani, NL o I-I 3, Allg. Korr. Die Präsenz vor Gericht übernahm Christian Raabe. Vertreten wurden fünf Nebenkläger gegen alle Angeklagte, von denen vier bereits im ersten Verfahren vertreten wurden; eine fünfte, polnische Nebenklägerin vertrat Raabe auf ausdrückliche Bitte Sehns. Die Liste der Nebenkläger in NL OI-I3.

I07 Urteil des Obersten Polnischen Gerichts vom 7.I0.1953 -II Kn 4I4/53. Die Verurteilung erfolgte wegen Zugehörigkeit zur SS als einer »verbrecherischen Organisation, Verantwortlichkeit für die im KZ Auschwitz herrschenden unmenschlichen Zustände [...], Mitwirkung bei der Aneignung des Eigentums der Häftlinge [...] zit. n. Urteil 4 Ks 3/63 v. I6.09.1966, Exemplar NL o I-I 4.

I08 Ebd. S. I4.

I09 Ebd. S. 34.

I I R Rabe, Plädoyer, S. 20 f. griff hier u.a. Dienstzeugnis zurück, in welchem Burger attestiert wurde, er habe es verstanden, »einmal von ihm als richtig erkannte Interessen [...] schonungslos wahrzunehmen « I I I Urteilsbegründung, S. 90 und S.92. 

im Häftlingskrankenbau und im Lagerbereich; ${ }^{112}$ damit habe er die »unter anderem von Hitler und Himmler begangenen Haupttaten $\left[. . . \ll_{\ll^{1}{ }^{13}}\right.$ gefördert. Mit dieser durchaus nicht unüblichen Urteilsbegründung verurteilte das Gericht Neubert wegen Beihilfe zum gemeinschaftlichen Mord zu 3 Jahren und 6 Monaten Zuchthaus unter Anrechnung der Untersuchungshaft. ${ }^{\text {II }} 4$

Kauls Opfer-Repräsentanz reichte hier durch die Vertretung von Nebenklägern aus Polen und der CSSR ${ }^{\text {IIs }}$ über die innerdeutsche Dimension hinaus. Die Bekundungen des Lagerarztes Fischer, selbst in einem Verfahren mit »zum Teil identischem Tatkomplex «I6 in der DDR angeklagt, trugen maßgeblich zum Nachweis der Gasbestellungen durch Burger und zur Selektionstätigkeit Erbers bei. ${ }^{17}$ Aufgrund des Anknüpfungspunktes Buna-Monowitz, des parallel laufenden »DDR-Konkurrenz-Verfahrens « und der Verzahnung beider Prozesse durch die Zeugenbenennung Fischers ist der Systemkonflikt hier zwar ebenso präsent wie im ersten Prozess, nicht zuletzt wegen der mangelnden medialen Aufmerksamkeit für das Verfahren jedoch nicht von größerer Breitenwirkung.

\section{Die Rechtsauffassung der Nebenklage}

Die Staatsanwaltschaft vertrat in beiden Auschwitz-Prozessen - im Sinne Fritz Bauers $^{\text {I } 8}$ - die Auffassung, die jeweiligen Handlungen der Angeklagten seien als natürliche Handlungseinheit im Sinne $\int 73$ StGB zu betrachten: »Die Angeklagten arbeiteten auf der Grundlage ihrer Anwesenheit in Auschwitz [...] und ernstlich unwidersprochen gebliebenen Einschaltung in das dortige Geschehen, [...] sämtlich am Fließband der Todesmaschinerie Auschwitz. Das [...] Gesamtverhalten der Angeklagten in Auschwitz [...] bildet eine natürliche Handlungseinheit. «II9 Dieser auch von Kaul vertretenen Auffassung trat Raabe noch im I. Prozess entgegen und plädierte unter Bezugnahme auf die Rechtsprechung des Bundesgerichtshofs, ${ }^{120}$ jede einzelne Tat in Auschwitz - so z.B. jede Selektion sei rechtlich gesondert, nach dem Prinzip der Tatmehrheit zu bewerten. Obgleich Raabe bereits im I. Auschwitz-Prozess zugestand, »dass zwar die Theorie der natürlichen Handlungseinheit den tatsächlichen Verhältnissen in Auschwitz mehr gerecht « ${ }^{\mathrm{I} 2 \mathrm{I}}$ werde, ging er davon aus, »dass eine gerechte Abgrenzung der Schuld des einzelnen Angeklagten lediglich aufgrund seiner Funktion im Lager [...] nicht möglich « sei und »die bloße Anwesenheit eines Angeklagten in Auschwitz [...] für eine Verurteilung « ${ }^{122}$ nicht ausreiche. Die Erfahrungen im ersten Krumey/Hunsche-Verfahren, die Erkenntnisse, die sich aus einer Abkoppelung der Tätigkeit des SEK vom Vernichtungsgeschehen

\footnotetext{
I 2 Urteilsbegründung, S. Iorf. und Ios.

I 3 Ebd. S. I 54 .

I 4 Ebd. Raabe ging ebenso von Beihilfe aus, forderte allerdings is Jahre Haft.. Raabe, Plädoyer, S. 22.

i I $\mathrm{Vgl}$. hierzu Rosskopf, Kaul (Fn. 59), S. 272.

I 6 Rosskopf, Kaul (Fn. 59), S. 273; zu dem Gesamtkomplex ebd., S. 273 ff.

I 17 Vgl. Urteilsbegründung S. 2 I.

I 8 Fritz Bauer, Ideal- oder Realkonkurrenz bei nationalsozialistischen Verbrechen?, Juristenzeitung, I967, S. 625-628; s. hierzu auch Friedrich Hoffmann, Die Rechtsauffassung der Staatsanwaltschaft im Auschwitz-Prozess, in: Auschwitz-Prozess, DVD-ROM (Fn. 30), S. 1.036 ff.

I 9 Plädoyer des OStA Großmann, 07.05.1965, 4 Ks 2/63, Auschwitz-Prozess, DVD-ROM (Fn. 30), S. 32.853 , AP 367.017 .

I 20 Christian Raabe, Plädoyer im ersten Frankfurter Auschwitz-Prozess, 2 1.05.1965, Auschwitz-Prozess, DVD-ROM (Fn. 30), S. 33.946ff.

I 2 I Ebd. 33.950.

I 22 Ebd. 33.945.
} 
durch eine allzu enge Tateinheitsdefinition ergaben, die Verzerrungen, die sich hierdurch in der Abbildung des Vernichtungskontexts zeigten, ließen die Frankfurter Nebenklage im zweiten Auschwitz-Prozess der wiederum von Tateinheit ausgehenden Staatsanwaltschaft folgen: ${ }^{123}$ »Hätte dagegen Hunsche beispielsweise eine viel geringere Rolle im Kommando gespielt, und hätte man ihm jedoch nachweisen können, dass er einmal eine ganz bestimmte Deportationsanweisung zum Bahnhof [...] gebracht hätte, so wäre er mit Sicherheit verurteilt worden.« Man könne »dem Phänomen der Massenmorde im Dritten Reich rechtlich nicht zufriedenstellend beikommen [...], wenn man das Geschehen in lauter einzelne, voneinander unabhängige Tathandlungen « ${ }^{\mathrm{I} 24}$ aufsplittere; hiermit formulierte die Frankfurter Nebenklage einen ähnlich weit ausgreifenden Tateinheitsbegriff - und damit auch eine Kritik an einem enger gefassten - wie der hessische Generalstaatsanwalt, der im ersten Frankfurter Auschwitz-Prozesses bereits eine Entschärfung des totalen Geschehens durch dessen Auflösung in Episoden gesehen hatte. «I25 Die Frankfurter Nebenklage relativierte ihre Rechtsposition über einen komplementären Tatkomplex des Vernichtungszusammenhangs, der Gettoisierung und Deportation, und übertrug diese relativierte Position im zweiten Auschwitz Prozess auf die Verwaltungskomponente des Lagers. So sei Burger als Leiter der Verwaltung »mit seiner gesamten dienstlichen Tätigkeit eine der Säulen [gewesen], die den ganzen Vernichtungsvorgang trugen. « »eine Gesamttätigkeit zur Aufrechterhaltung dieser mörderischen Ordnung [überwiege] bei weitem die Bedeutung seiner Selektionstätigkeit.«Tatmehrheit hingegen sah Raabe nunmehr lediglich bei sogenannten Exzesstätern gegeben, die über das "geforderte Soll hinaus Menschen aus eigener Initiative ermordeten. ${ }^{126}$ Betrachtete die Frankfurter Nebenklage den ganzen Vernichtungskomplex als Rahmen für eine Handlung, fasste das Schwurgericht, auch von Tateinheit ausgehend, diesen in seiner Urteilsbegründung enger. Es interpretierte jede einzelne Vernichtungsaktion als eine Handlung, an der Burger durch die Gasbestellungen mitgewirkt habe, und nahm somit lediglich ein Fragment der Tätigkeit des Leiters der Verwaltung in den Blick. Eine ähnlich enge Fokussierung hatte das Schwurgericht im ersten Auschwitz-Prozess vorgenommen: Wenngleich das Gericht die »Endlösung « auf einen Willensentschluss Hitlers zurückführte, wertete es den Gesamtvorgang der Vernichtung dennoch nicht als eine Handlung, sondern sah den Beginn einer einheitlichen Tat in der Auslösung der jeweiligen Vernichtungsaktion, einheitliche Handlungen sah das Gericht im jeweiligen Prozedere von der Selektion bis zum Vergasungsvorgang gegeben. ${ }^{127}$ Als wesentliches Kriterium bei der Ahndung an unterschiedlicher Stelle im Vernichtungszusammenhang stehender Tatkomplexe erweist sich somit die Weite bzw. die Enge der Definition einer einheitlichen Handlung - mit

I 23 Begründung: Raabe, Plädoyer im zweiten Auschwitz-Prozess, S. 6.

I 24 Beide Zitate ebd., Plädoyer, S. 6.

I 25 Bauer, Im Namen des Volkes (Fn. 2I), S. 83. Vgl. auch Irmtrud Wojak, Die Verschmelzung von Geschichte und Kriminologie. Historische Gutachten im ersten Frankfurter Auschwitz-Prozess, in: Norbert Frei u.a. (Hrsg.), Geschichte vor Gericht, Historiker, Richter und die Suche nach Gerechtigkeit, München 2000, S. 29-45. In gleicher Front Robert M.W. Kempner rückblickend auf den zweiten Krumey/Hunsche-Prozess: Die juristische Schuld der Angeklagten könne nur angemessen festgestellt werden, wenn das gesamte damalige Zeitgemälde geschaffen werde. »Alles aus dem Zusammenhang Gerissene endet in einer schiefen juristischen Beurteilung, die nicht dem wirklichen Recht entspricht.« Robert M.W. Kempner, Lebenslänglich und zwölf Jahre Zuchthaus wegen Ermordung der ungarischen Juden, in: Illustrierte Neue Welt 9/1969.

I 26 Beide Zitate Raabe, Plädoyer im zweiten Auschwitz-Prozess, S.8.

I 27 Urteilsbegründung $4 \mathrm{Ks} 2 / 63$, S. I $14 \mathrm{f}$. 
führung der Tat gewinnt diese Frage freilich an Relevanz.

Raabe wandte sich entschieden gegen eine nicht nur dem Urteil des ersten Auschwitz-Prozesses zugrundegelegte Interpretation, »Hitler, Himmler und Heydrich seien die Haupttäter gewesen, die übrigen Tatbeteiligten [...] dagegen im allgemeinen Gehilfen «, die zu einer »vom Gesetzgeber und der Rechtsprechung sicherlich nicht gewollten Verschiebung der Gewichte führe. « ${ }^{\mathrm{I} 28}$ Die Frankfurter Nebenklage anerkannte als Kriterium zur Abgrenzung zwischen Täterschaft und Beihilfe ausdrücklich die subjektive Teilnahmetheorie ${ }^{\text {I29 }}$ und zeigte sich in den Komplementärverfahren als eine Korrektivinstanz hinsichtlich ihrer allzu begrenzten Fokussierung sowie ihrer extensiven Auslegung im Zuge der Tendenz, Täter von nationalsozialistischen Gewaltverbrechen als Gehilfen zu judizieren.

Eine strafprozessuale, eine prozessatmosphärische und eine über die Verfahren hinaus in den gesellschaftspolitischen Raum greifende Wirkung erweisen sich als Eckpfeiler der Nebenklagekonzeption - den jenseits des Strafprozessualen liegenden Aspekten kommt hierbei ebenso große Bedeutung zu wie dem im Namen der Opfer erhobenen Strafanspruch. Während Ormonds Schwerpunkte bei den historischen und politischen Aspekten des Gesamtkomplexes Auschwitz und einer über das Prozessgeschehen hinausreichenden Aufklärungsintention liegen, bleiben Raabes Aktivitäten mehr auf die juristische Dimension des Verfahrens konzentriert. Als Korrektivinstanz in den Verfahren zeigt sich die Frankfurter Nebenklage gegen eine Segmentierung der zur Verhandlung stehenden Tatkomplexe, indem (auch) die Nebenklage den Vernichtungszusammenhang in den Verfahren präsent werden ließ. In diesem Zusammenhang arbeitet sie gleichsam als Verstärker für die Grundintentionen des hessischen Generalstaatsanwalts Fritz Bauer. Als Korrektiv tritt sie überdies in Erscheinung gegen eine Instrumentalisierung der Opfer und des Verfahrensgegenstandes in der Systemauseinandersetzung, aber auch als Korrektiv zu der Tendenz in Verfahren über nationalsozialistische Gewaltverbrechen Angeklagte als Gehilfen zu qualifizieren. Hiermit leistet die Nebenklage an der Seite der Staatsanwaltschaft einen bedeutenden, über ihre Opferschutzfunktion hinausgehenden Beitrag zur Wahrheitsfindung, greift aber auch aus in einen gesellschaftlichen und politischen Raum.

"Es ist mir eine Genugtuung «, schrieb der von Ormond und Raabe vertretene Nebenkläger Werner Krumme am 30.8.1966 an die Kanzlei, »mit welchem Nachdruck und mit welch vorzüglicher Begründung Sie [...] Ihre Einsprüche erhoben haben. Die Interessen der Auschwitzer liegen bei Ihnen wirklich in den besten Händen. «130 Mit dem Begriff »Genugtuung « ist freilich ein Zeugnis ausgestellt.

\section{Summary}

Im ersten Frankfurter Auschwitz-Prozess (1963-1965) standen vorrangig Tatbeteiligte vor Gericht, die im Vernichtungsprozess an letzter Stelle standen, den

I 28 Zusammenfassend zit. n. Raabe Plädoyer, S. I7 und S. i 8.

I 29 Raabe ging davon aus, dass »die Scheidung von Täterschaft und Beihilfe aufgrund rein objektiver Merkmale nicht möglich « sei - die subjektive Teilnahmetheorie sei die »einzig praktikable subjektive Abgrenzungstheorie.«ebd. S. I6

I 30 Angesichts dessen Bemühungen, der Haftverschonung eines Angeklagten entgegenzuwirken. Schreiben Krumme an Raabe vom 30.8.1966, in NL OI-I 2. 
eigentlichen Tötungsvorgang ausführten oder Selektionen vornabmen. Mit dem zweiten Verfabren (1966) rückt nun entsprechend der früheren Funktion der hier Angeklagten die Verwaltungskomponente des Vernichtungszusammenhangs ins Blickfeld. Ein weiterer, das Frankfurter Landgericht 13 Jahre beschäftigender Verfahrenskomplex (1956-1969) gegen Hermann Krumey und Otto Hunsche, "Mitarbeiter" in Eichmanns Sondereinsatzkommando in Ungarn an maßgeblicher Stelle, fokussiert schließlich den Aspekt der Deportation. An diesen hinsichtlich ibrer Tatkomplexe komplementären Verfabren beteiligen sich VerfolgtenOrganisationen über das Instrument der Nebenklage. Als Eckpfeiler dieser von den Frankfurter Anwälten Henry Ormond und Christian Raabe übernommenen Nebenklagevertretung zeigen sich - neben dem im Namen der Opfer erbobenen Strafanspruch - eine über den strafprozessualen Rabmen hinausgreifende gesellschaftliche Aufklärungsintention sowie eine prozessatmosphärische Komponente, das Bestreben, die Realität Auschwitz im Verfahren präsent werden zu lassen. Die Frankfurter Nebenklage leistete an der Seite der Staatsanwaltschaft einen nicht unwesentlichen Beiträge zur Wabrheitsfindung und tritt - im Sinne des hessischen Generalstaatsanwalts Fritz Bawer - als Korrektivinstanz in Erscheinung gegen eine Segmentierung der zur Verhandlung stehenden Tatkomplexe, aber auch zu der Tendenz, NS-Täter als Gebilfen zu judizieren. Vor dem Hintergrund der Ost-West-Polarisierung tritt sie überdies als Korrektiv auf gegen eine Instrumentalisierung der Opfer und des Verfahrensgegenstandes in der innerdeutschen Systemauseinandersetzung um den Umgang mit der NS-Vergangenheit.

\section{Studien und Materialien zur Verfassungsgerichtsbarkeit}

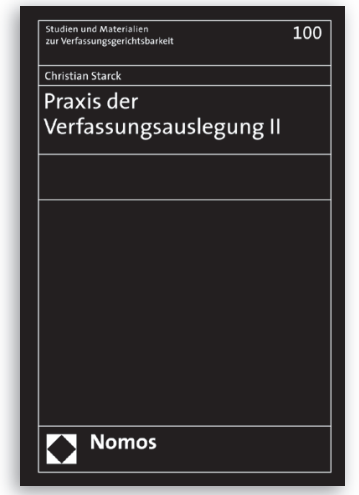

Praxis der Verfassungsauslegung II

Von Prof. Dr. Christian Starck, Universität Göttingen 2007, 288 S., geb., 69,-€, ISBN 978-3-8329-2152-1 (Studien und Materialien zur Verfassungsgerichtsbarkeit, $B d .100)$

Bitte bestellen Sie bei Ihrer Buchhandlung oder bei Nomos 07221/2104-37| 产-43| www.nomos.de | sabine.horn@nomos.de

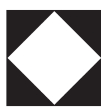

Nomos 